ARTICLE

\title{
HOXA13 in etiology and oncogenic potential of Barrett's esophagus
}

Vincent T. Janmaat (10 ${ }^{1}$, Kateryna Nesteruk (D) ${ }^{1}$, Manon C. W. Spaander ${ }^{1}$, Auke P. Verhaar ${ }^{1}$, Bingting Yu ${ }^{1}$, Rodrigo A. Silva (1) 1, Wayne A. Phillips (1) 2,3,4, Marcin Magierowski (10 1,5, Anouk van de Winkel', H. Scott Stadler ${ }^{6}$, Tatiana Sandoval-Guzmán (10 ${ }^{7}$, Luc J. W. van der Laan (i) ${ }^{8}$, Ernst J. Kuipers (i) ${ }^{1}$, Ron Smits (iD ${ }^{1}$, Marco J. Bruno (i) 1, Gwenny M. Fuhler ${ }^{1}$, Nicholas J. Clemons (id ${ }^{2,3} \&$ Maikel P. Peppelenbosch (D) ${ }^{1 凶}$

Barrett's esophagus in gastrointestinal reflux patients constitutes a columnar epithelium with distal characteristics, prone to progress to esophageal adenocarcinoma. HOX genes are known mediators of position-dependent morphology. Here we show HOX collinearity in the adult gut while Barrett's esophagus shows high HOXA13 expression in stem cells and their progeny. HOXA13 overexpression appears sufficient to explain both the phenotype (through downregulation of the epidermal differentiation complex) and the oncogenic potential of Barrett's esophagus. Intriguingly, employing a mouse model that contains a reporter coupled to the HOXA13 promotor we identify single HOXA13-positive cells distally from the physiological esophagus, which is mirrored in human physiology, but increased in Barrett's esophagus. Additionally, we observe that HOXA13 expression confers a competitive advantage to cells. We thus propose that Barrett's esophagus and associated esophageal adenocarcinoma is the consequence of expansion of this gastro-esophageal HOXA13-expressing compartment following epithelial injury.

\footnotetext{
${ }^{1}$ Department of Gastroenterology and Hepatology, Erasmus MC - University Medical Center Rotterdam, Rotterdam, The Netherlands. ${ }^{2}$ Division of Cancer Research, Peter MacCallum Cancer Centre, Melbourne, VIC, Australia. ${ }^{3}$ Sir Peter MacCallum Department of Oncology, The University of Melbourne, Melbourne, VIC, Australia. ${ }^{4}$ Department of Surgery (St. Vincent's Hospital), The University of Melbourne, Melbourne, VIC, Australia. ${ }^{5}$ Department of Physiology, Faculty of Medicine, Jagiellonian University Medical College, Cracow, Poland. ${ }^{6}$ Department of Skeletal Biology, Shriners Hospital for Children, Portland, OR, USA. ${ }^{7}$ DFG-Center for Regenerative Therapies, Technische Universität Dresden, Dresden, Germany. ${ }^{8}$ Department of Surgery, Erasmus MC University Medical Center Rotterdam, Rotterdam, The Netherlands. Share senior authorship: Gwenny M. Fuhler, Nicholas J. Clemons \& Maikel P.

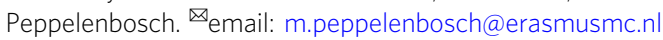


B arrett's esophagus (BE) and gastric intestinal metaplasia (IM) are important risk factors for adenocarcinoma of the esophagus and stomach. In the esophagus, the chronic inflammation associated with gastroesophageal reflux disease (GERD) is believed to lead to Barrett's esophagus (BE), a cryptstructured columnar epithelium with distal gastrointestinal (GI)tract characteristics, located just above the gastro-esophageal junction (GEJ). BE is a precursor lesion for esophageal adenocarcinoma $(\mathrm{EAC})^{1,2}$, a disease which has shown a strong increase in incidence in the past decades. Analogously, H. pylori-infection can degenerate into atrophic gastritis and gastric IM, which in turn can progress into gastric cancer, the third leading cause of cancer-related death ${ }^{3}$. Similarly, while absolute risk is low, heterotopic tissues in Meckel's diverticula and gastric inlet patches of the proximal esophagus represent relatively high-risk regions for adenocarcinoma comparatively to other sites of the ileum and proximal esophagus, respectively ${ }^{4,5}$. Therefore, a deeper understanding of the biology of $\mathrm{BE}$ and gastric IM is necessary for designing rational avenues for the prevention and treatment of GI cancers.

$\mathrm{BE}$ is characterized by the presence of cells with a caudal intestinal phenotype at a rostral location. Therefore, dysregulation of positional specification is likely involved in the etiology of BE. Regulation of rostral-caudal patterning of specialized tissue in embryology and adulthood is to a large extent dependent on the concerted action of two evolutionary highly conserved gene systems, the Caudal-related Homeobox $(C D X)$ transcription factor gene family and the genes of the Homeobox (HOX) cluster. A substantial research effort has been invested in investigating the role of $C D X$ genes in positional misspecification in $\mathrm{BE}^{6}$. However, these efforts have not yielded convincing evidence that these genes are the principal mediators of the distal phenotype in this disease $e^{7,8}$. Intriguingly, however, a microarray-based gene expression study of $\mathrm{BE}$ suggested potential misregulation of the $H O X$ gene family in $\mathrm{BE}^{9}$. HOX genes are linked to morphological transformations and neoplasia ${ }^{10,11}$. Four clusters of $H O X$ genes, HOXA to HOXD, have been defined. The $3^{\prime}$ to $5^{\prime}$ sequence of $H O X$ gene paralogues corresponds to the sequence in which they act along the rostrocaudal axis. This property is termed collinearity and links clustering to function. Previously, a Hox expression gradient was found along the murine embryonic gut ${ }^{12}$. Ectopic Hox expression in mice can alter intestinal differentiation ${ }^{8}$. A HOX gradient along the adult human gut has also been reported ${ }^{13}$, but that study involved pooling full thickness gut specimens, limiting data interpretation. Nevertheless, we feel that there is sufficient evidence to prompt exploring the function of $H O X$ gene expression with respect to positional identity in physiology and pathology of the GI-tract in general and in BE in particular.

Here, we show that single cells of the upper GI tract express the distal gene $H O X A 13$, that their number is upregulated in $\mathrm{BE}$ and that HOXA13 conveys phenotypic metaplasia and increases proliferation.

\section{Results}

HOX cluster gene expression in the GI tract is collinear in men and mice. Investigating $H O X$ gene mRNA expression in the murine and human gastrointestinal tract, we observed collinearity that is similar in adult humans and mice (Fig. 1a for human HOXA, Supplementary Fig. 1 for all HOX genes and Supplementary Fig. 2 for graphical presentation of the studied $H O X$ clusters and the locations of biopsies taken along the human $(n=$ 3 ) and mouse $(n=4)$ GI tract). The highest HOX gene cluster expression was observed in the colon, except for the HOXC cluster. For individual paralogues, there is a higher expression of $5^{\prime} H O X A / B$ genes in the distal GI-tract from $H O X A 5 / B 5$ onward.
Of all HOXA paralogues, expression of HOXA13 was highest and restricted to the colon (Fig. 1a). HOXA13 expression is regulated by LncRNA HOTTIP, which is located $5^{\prime}$ to HOXA13 14 . Accordingly, HOTTIP and HOXA13 share a similar expression pattern (Supplementary Fig. 1a). For $H O X D$, all paralogue genes have increased expression in the distal colon, while HOXC expression is mainly localized in the proximal and ileal regions. Thus, HOX gene expression is linked to positional identity in the mammalian gut, and collinearity is particularly strong for the $H O X A / B$ paralogues.

Subsequently, we addressed the question as to whether GI HOX coding is already present at the GI stem cell stage, or is established only upon the formation of differentiated derivatives. For this, we used publicly available data published by Wang et al. which contains the mRNA expression of human stem cells isolated from the GI-tract and either cultured as stem cells or differentiated in an air-liquid interface (ALI) ${ }^{15}$. Analysis of this data shows that HOX gene expression patterns in stem cell and ALI cultures are similar. HOXA and $B$ cluster genes have a significantly higher expression in the large intestine as compared to the small intestine, in particular $5^{\prime}$ HOXA genes including HOXA13 (Fig. 1d for HOXA genes, for clusters $H O X B, C$, and $D$ see Supplementary Fig. 3). No clear regulation of the HOXC or $D$ clusters is seen in this in dataset, with exception of an upregulation of $\mathrm{HOXC10}$ in the large intestine. Hence, $H O X$ coding is an inherent feature of the locationspecific stem cell and is maintained in its derivatives.

HOXA13 in BE, GI heterotopias and GI cancers. As positional phenotype is linked to HOX status in physiology, we subsequently characterized $H O X$ mRNA expression in several metaplastic tissues known to assume the morphological phenotype of other intestinal locations, as well as their sequelae. BE shows upregulation of $H O X A 10,11$, and 13, and $H O X B$ 6, 7, 9 , and 13 mRNA by qPCR when compared to the normal squamous esophagus (Fig. 1b and Supplementary Fig. 4), which closely resembles colonic $H O X A$ and $B$ expression patterns. High $5^{\prime}$ HOXA gene expression is also present in columnarlined esophagus without goblet cells (CLE; a BE-related condition), esophageal adenocarcinoma (EAC), and IM of the stomach (Fig. 1b, c). In accordance with a regulatory role for HOTTIP on HOXA13 expression, we find that HOTTIP is also overexpressed in $\mathrm{BE}$, and correlates with $H O X A 13$ expression patterns (Supplementary Fig. 5a-c). HOTAIR, a lncRNA located in the HOXC cluster and associated with chromatin reprogramming in cancer progression ${ }^{16}$ is upregulated as well (Supplementary Fig. $5 \mathrm{~d}-\mathrm{f})^{14}$. We concluded that BE, EAC and various metaplasias with caudal histo-morphological characteristics have $H O X A$ and $H O X B$ expression patterns typical of the caudal GI-tract, with upregulation of HOXA13 expression being the prominent feature. Heterotopias, namely the gastric inlet patch in the proximal esophagus and heterotopia of the Meckel's diverticulum, are tissues which have a physiological appearance, but are normally found in a different location. Both these heterotopias are characterized by abundant HOXA13 mRNA expression (Fig. 1c), although intriguingly the direction of epithelial metaplasia for Meckel's diverticulum is of an anterior rather than posterior phenotype, indicating an exception from the pattern in case of Meckel's diverticulum. One of the existing hypotheses on the cell of origin of $\mathrm{BE}$ states that $\mathrm{BE}$ may arise from cells with progenitor properties that are able to give rise to a variety of cell types ${ }^{17}$. To investigate whether aberrant $\mathrm{HOX}$ gene expression in $\mathrm{BE}$ is established at the level of the epithelium-specific stem cell, we interrogated the publically available data of Yamamoto 
a

Pathology:

$\begin{array}{lllllllll}\text { squamous esophagus of BE patient } & 3 & 0 & 1 & 1 & 3 & 4 & 11\end{array}$

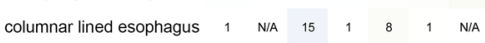

$\begin{array}{lllllllll}\text { Barrett's metaplasia } & 1 & 0 & 1 & 0 & 1 & 3 & 1\end{array}$

$\begin{array}{llllllll}\text { esophageal adenocarcinoma } & 2 & 0 & 0 & 1 & 4 & 9 & 10\end{array}$

$\begin{array}{lllllllll}\text { gastric intestinal metaplasia } & 2 & \text { N/A } & 7 & 1 & 8 & 3 & 1\end{array}$

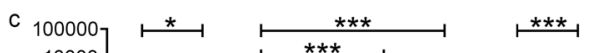

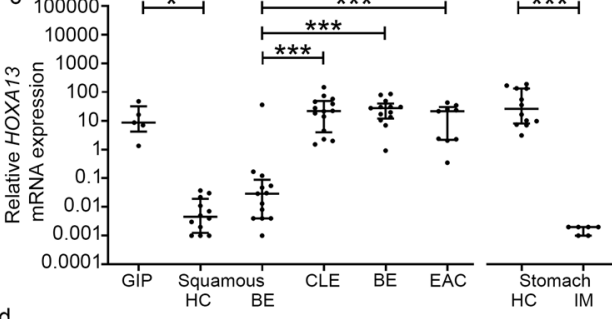

duodenum $\begin{array}{lllllll}1 & 1 & 2 & 2 & 1 & 0 & 0\end{array}$

$\begin{array}{llllllll}\text { distal ileum } & 4 & 3 & 2 & 0 & 6 & 17 & 10\end{array}$

$\begin{array}{cccccccc}\text { colon ascendens } & 3 & 2 & 4 & 0 & 8 & 22 & 20 \\ & & 4 & 3 & 0 & 15 & 44 & 36\end{array}$

rectum $\begin{array}{llllllll}3 & 2 & 2 & 0 & 6 & 14 & 15\end{array}$
$9 \quad 10 \quad 11 \quad 12 \quad 13 \quad e$

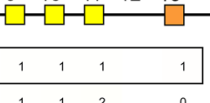

$\begin{array}{llllllll}\text { proximal ileum } & 5 & 1 & 1 & 3 & 2 & 1 & 6\end{array}$

\begin{tabular}{ccc}
1 & 1 & 2 \\
1 & 1 & 0 \\
3 & 4 & 17 \\
21 & 11 & 1 \\
\hline 310 & 281 & 139 \\
406 & 279 & 3626 \\
532 & 290 & 3709 \\
178 & 328 & 1700
\end{tabular}

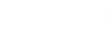

$\begin{array}{lll}13 & 6 & 0\end{array}$

$20 \quad 98$ NA

$16 \quad 116 \quad 2422$

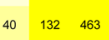

7. $43 \quad 4333$

.$* * *$

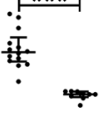

苞

Barrett's vs squamous esophagus
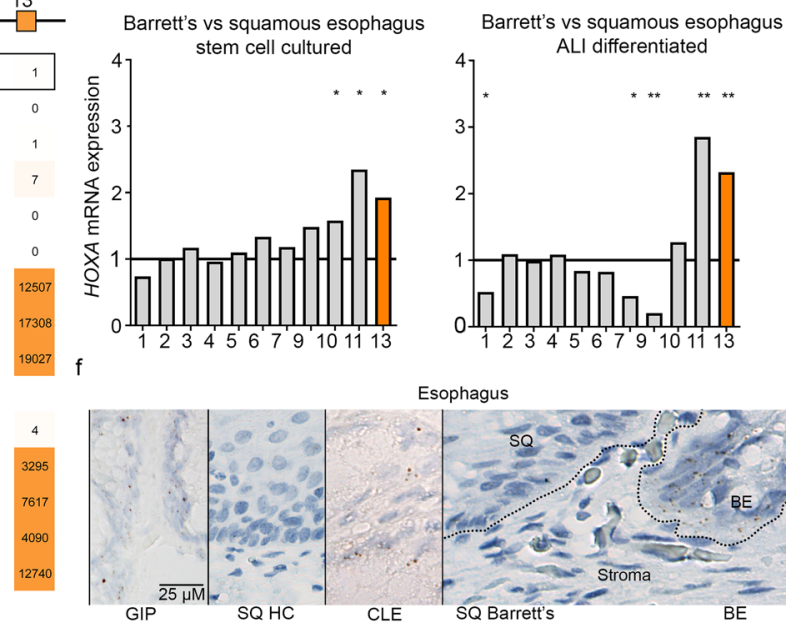

Esophagus
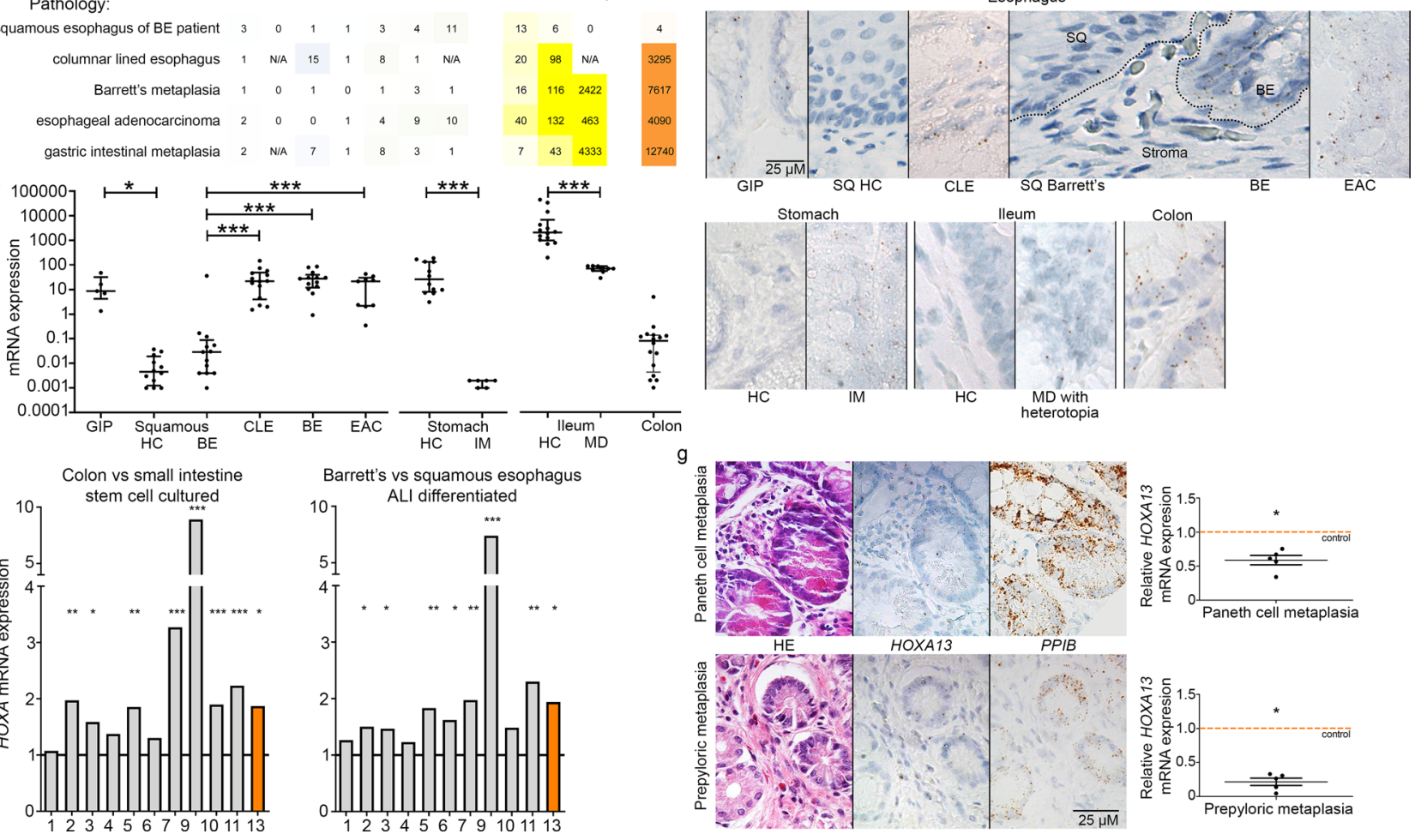

Fig. 1 HOXA cluster gene expression shows collinearity along the adult gastrointestinal tract but is deregulated in Barrett's esophagus (BE), various metaplasias and esophageal adenocarcinoma (EAC). a HOXA cluster genes are collinearly expressed along the gastro-intestinal (GI)-tract of adult humans $(n=3)$. Numbers represent mRNA fold changes relative to the esophagus and thus can be compared within each HOXA paralogue member but not between them. b HOXA cluster gene expression in the squamous esophagus of BE patients $(n=13)$, columnar lined esophagus (CLE) ( $n=14)$, BE ( $n=$ $13)$, EAC $(n=12)$, and gastric intestinal metaplasia $(I M)(n=12)$ is characterized by an upregulation of $5^{\prime}$ HOXA genes. Numbers represent mRNA fold changes relative to the esophagus of healthy individuals. c HOXA13 expression quantified by QPCR in BE, CLE, IM of the stomach, and heterotopias along the GI tract with their corresponding physiological epithelia. Squamous epithelium (SQ) Barrett's and BE are derived from the same person ( $n=13)$. Gastric inlet patch (GIP; $n=5)$; healthy control (HC) squamous esophagus $(n=12) ; \operatorname{CLE}(n=14) ; \operatorname{BE}(n=13)$; $\operatorname{EAC}(n=12)$; stomach $(n=14) ;$ gastric IM ( $n=12)$; ileum $(n=6)$; Meckel's diverticulum (MD) with gastric heterotopia $(n=14)$, and colon $(n=9)$. Median $\pm I Q R,{ }^{\star} p<0.05 ;{ }^{\star \star} p<0.01$; ${ }^{\star \star \star} p<0.001$. For esophagus, Kruskal-Wallis test with Dunn's multiple comparisons test (SQ healthy vs. GIP, $p=0.015$; SQ healthy vs. CLE, $p<0.0001 ; \mathrm{SQ}$ healthy vs. BE, $p$ $<0.0001$; SQ healthy vs. EAC, $p=0.0009$ ). For stomach and ileum, Mann-Whitney test (two-tailed), $p<0.0001$. d HOXA cluster genes, in particular $5^{\prime}$ HOXA genes including HOXA13, have a higher expression in the large intestine ( $n=3$ in technical duplicate) compared to the small intestine ( $n=3$ in technical duplicate), in both stem cells (left panel) and differentiated cells (right panel). Normalization was performed by setting mRNA expression to 1 for the small intestine. ${ }^{\star} p<0.05 ;{ }^{\star \star} p<0.01 ;{ }^{\star \star \star} p<0.001$. This figure includes no estimate of variance as the empirical Bayes-moderated two-sided t-statistic was used which does not generate a standard error. e $5^{\prime}$ HOXA cluster gene expression in BE is higher compared to the squamous esophagus in stem cell and air-liquid interface (ALI) differentiated cultures. $n=12$ (BE) versus $n=2$ (squamous esophagus) in technical duplicates are depicted for stem cell cultures and $n=1$ each for ALI differentiated samples in technical duplicates. Normalization was performed by setting mRNA expression to squamous esophagus. ${ }^{\star} p<0.05 ;{ }^{\star \star} p<0.01 ;{ }^{\star \star \star} p<0.001$. This figure includes no estimate of variance as the empirical Bayes-moderated two-sided t-statistic was used which does not generate a standard error. $\mathbf{f}$ Deregulation of HOXA13 expression in gastrointestinal tract pathology as evaluated with RNA in situ hybridization in clinical samples. HOXA13 is upregulated in IM and heterotopia and downregulated in pyloric and Paneth cell metaplasia in the colon. One sample of each tissue type was analyzed. $\mathbf{g}$ Downregulation of HOXA13 expression (corrected for peptidylprolyl isomerase B - PPIB expression) relative to adjacent non-metaplastic tissue, was observed for Paneth cell metaplasia $(n=5 ;$ FC $0.59 ; p=0.0003)$ and pyloric metaplasia $(n=5 ;$ FC $0.22 ; p=0.0001)$ (lower panels). Unpaired t-test (two-tailed). Representative images of hematoxylin and eosin (HE) staining, HOXA13 RNA-scope, and PPIB reference gene RNA-scope of Paneth cell metaplasia (from the colon) present in two glands to the bottom right (upper panels) and pyloric metaplasia (from the colon) in the top left two glands (middle panels) are shown. 
et al. ${ }^{15,18}$. HOX gene expression patterns in squamous esophageal and BE stem cells as well as their respective ALIdifferentiated derivatives were retrieved. HOX gene expression in stem cell cultures from these locations is similar to their ALI differentiated counterparts (Fig. 1e, Supplementary Fig. 3). In $\mathrm{BE}$ stem cells, an upregulation of $5^{\prime} \mathrm{HOXA}$ genes (Fig. 1e) as well as $\mathrm{HOXB6}_{1}, 7,13$, and $\mathrm{HOXC10}$ is seen (Supplementary Fig. 3), reaching levels similar to those observed in the colon. Thus, alternative HOX coding associated with BE is established at the epithelium-specific stem cell level and is maintained in derivatives of the stem cells involved.

According to the collinearity theory, a paralogue group 13 member is more likely to confer the distal characteristics seen in $\mathrm{BE}$ as compared to more anterior paralogue group members ${ }^{19}$. Of the paralogue group 13 members, HOXA13 and HOXB13 are overexpressed in $\mathrm{BE}$, with HOXA13 showing much higher expression compared to HOXB13 in BE, EAC, and IM of the stomach (Supplementary Fig. 4). Therefore, while HOX genes such as $H O X A 11, B 6, B 9$, and $B 13$ are also potentially interesting candidates, here we chose to focus on the HOXA13 gene for further in-depth analysis of different metaplastic tissues. As immunohistochemistry for HOXA13 was unsuccessful, (two antiHOXA13 antibodies were tested, but lacked specificity) we resorted to in situ hybridization (ISH) for HOXA13 to further confirm the observed atypical expression of this gene in different tissues (examples shown in Fig. 1f). Metaplasia is found throughout the GI-tract. While BE and IM acquire a more distal phenotype, distally located colonic pyloric and Paneth cell metaplasia, related to inflammatory bowel disease, acquire a more rostral phenotype ${ }^{20}$. Accordingly, downregulation of HOXA13 expression (corrected for PPIB expression as a reference gene) relative to adjacent non-metaplastic tissue, was seen for these tissues (Fig. 1g, again supporting a role for HOXA13 in positional identity).

Binary regulation of $\mathrm{HOXA13}$ expression. To study in more detail which of the cells in the healthy GI-tract express Hoxa13, we employed a murine model in which the endogenous mouse Hoxa13 promoter drives the expression of a Hoxa13-GFP fusion protein. Within the epithelial compartment, the proximal expression border is located at the transition from the distal to the proximal colon as can be seen from fluorescent images and images of anti-GFP IHC staining (Fig. 2a, b and Supplementary Fig. 6a-d for bigger overview images). This proximal expression border seems to be crypt-clonal, with some crypts expressing Hoxa13 and others not (see arrows in Fig. $2 b$ and close-up in Fig. 2c). Functional consequences of this clonality are unknown and, while beyond the scope of the present manuscript, present an interesting biological question. The distal Hoxa13-GFP expression is limited by the anal squamocolumnar junction (SC); Supplementary Fig. 6e, please note this cannot be appreciated in Fig. 2a, as this part was damaged for this mouse). To investigate whether these local gradients of Hoxa13 expression are also present in humans, HOXA13 mRNA expression was assessed by qPCR in an additional set of biopsies taken from different colonic locations. Cecal biopsies are HOXA13 negative, while HOXA13 expression increases from the ileocecal valve to the distal transverse colon, demonstrating a similar expression pattern as observed in the mouse (Fig. 2d).

In addition to a Hoxa13 gradient along the GI tract, epithelial Hoxa13-GFP expression is also tightly regulated along the basoluminal axis of individual crypts. Proximally, only apical expression is seen, while distally Hoxa13-GFP is expressed along the entire baso-luminal axis of the crypts (Fig. 2e). In addition, mesenchymal expression is observed in the cells just beneath the epithelium in the proximal colon (Fig. 2e). Within the cell, the strongest signal is co-localized with nuclei, as expected, but cytoplasmic staining is also seen which can be explained by ribosomal synthesis (Fig. 2e).

We concluded that spatial regulation of HOXA13 expression is very precise, robust and colon-specific, raising questions as to the cellular origin of the HOXA13 expression observed in BE.

Individual Hoxa13/HOXA13-positive cells in the upper GI tract. No significant expression of HOXA13 mRNA was seen in the squamous esophagus of BE patients by qPCR (Fig. 1c, e), suggesting that GERD does not provoke HOXA13 expression per se. Indeed, when two primary immortalized squamous esophageal cell lines (EPC2-hTERT and HET-1A) were exposed to either bile or acid, only minor effects on HOXA13 expression were observed (two to fourfold from a low baseline expression; Fig. 3a, b), more in agreement with cells having a relatively high HOXA13 expression showing better survival of the treatment rather than upregulation of expression per se. This was confirmed by analysis of the publicly available single cell RNAseq database recently published by Owen et al. ${ }^{21}$. Results at single cell level demonstrate the presence of a small population of HOXA13positive cells in the normal squamous esophagus of BE patients (8\%). In BE tissue, the percentage of these HOXA13-positive cells increase to $30 \%$, but their individual HOXA13 mRNA levels are not increased as compared to HOXA13-expressing cells of the normal esophagus (Fig. 3c, d). Similarly, the number of HOXA13positive cells, but not HOXA13 expression per cell, is increased in IM of the stomach, early gastric cancer, and colorectal cancer (Fig. 3c, d). Thus, we further investigated Hoxa13 at the cellular level in our samples. Although HOXA13 mRNA expression was detectable in only one of four mice in the upper GI-tract by qPCR (Supplementary Fig. 2), detailed inspection of specimens involved did identify single Hoxa13-positive cells in the stomach of Hoxa13-GFP mice by immunohistochemistry. Such signal was present at the basolateral side along the stomach starting from the GEJ, but not seen in the squamous cells along the esophagus, nor the stroma (Fig. 4a and Supplementary Fig. 7). This is of particular interest as the GEJ has been suggested as a place of origin of $\mathrm{BE}^{17}$. A littermate negative for Hoxa13-GFP showed no positivity (Supplementary Fig. 6d, Supplementary Fig. 7d). Subsequently, we employed ISH for HOXA13 on surgical samples from the human GEJ of three adult patients to analyze the presence of HOXA13-expressing cells in the human upper GI tract. In all three specimens, the GE junction area contained a clear positive signal for HOXA13 mRNA, some signal was seen in cells of the proximal stomach, while signal was even lower in the squamous epithelium and stroma (Fig. 4b and Supplementary Fig. 8a, b). Esophageal submucosal glands $(\mathrm{ESMG})^{21}$ were present in one sample and were $H O X A 13$ positive (Fig. 4c). Interestingly, ESMG were also highly positive for $K R T 7^{+} K R 5^{+} \mathrm{TP} 63^{+}$cells (previously postulated as the cell of origin $\mathrm{BE}$ origin in $\mathrm{GEJ}^{22}$ ) although unlike $\mathrm{HOXA13}{ }^{+}$cells, $\mathrm{KRT} 7^{+} \mathrm{KR} 5^{+} \mathrm{TP} 63^{+}$triple positive cells were not identified in the stomach (Fig. 4d). (Of note, this is showed for one sample and we were unable to assess possible HOXA13 co-expression with these triple positive cells due to absence of specific HOXA13 antibodies). We also studied HOXA13 expression in the GEJ of three spontaneously aborted human fetuses of 17-20 weeks of age, a gestation period characterized by transition of the esophageal epithelium from columnar to a squamous phenotype. We observed high and specific HOXA13 expression at the gastric cardia, while more distal stomach and esophageal epithelium were less positive (Fig. 4e, Supplementary Fig. 8c, d). These data imply that HOXA13-positive cells are present in the human embryonic 


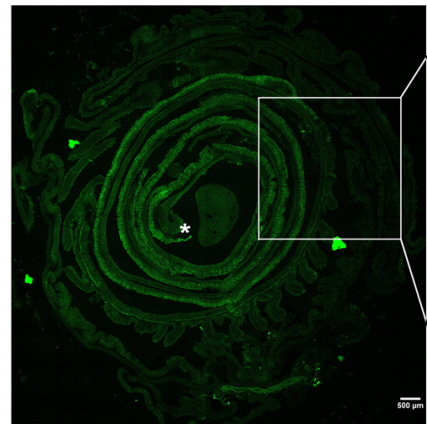

d

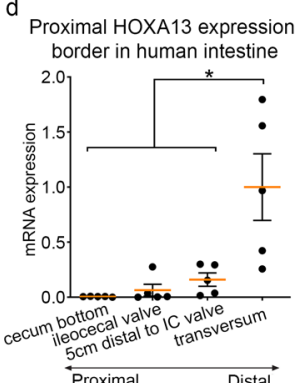

e $b$
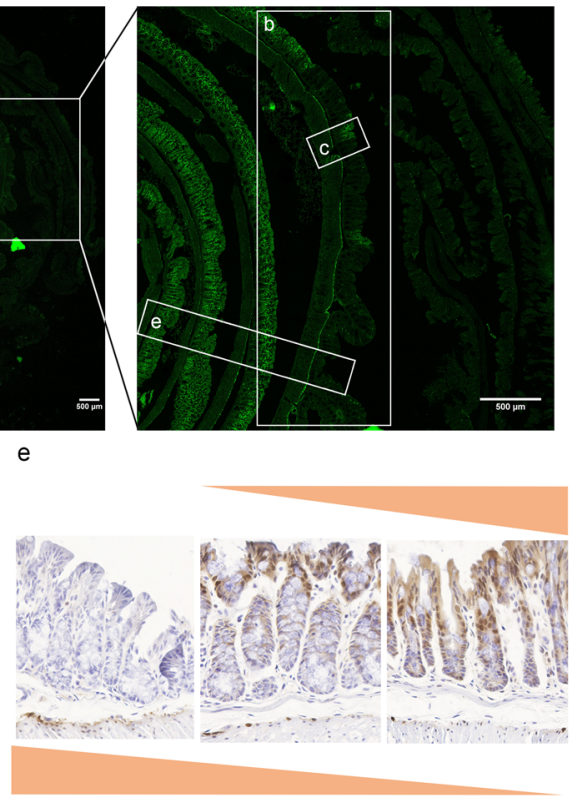

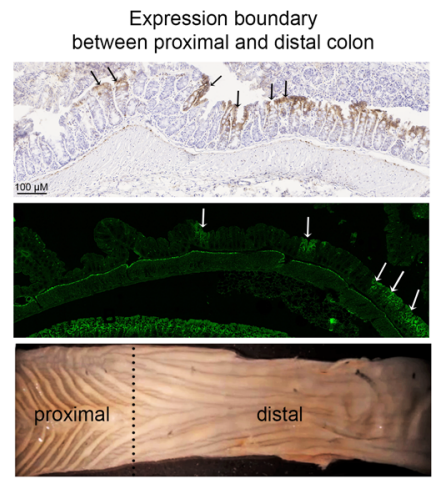

Crypt clonal and mesenchymal expression
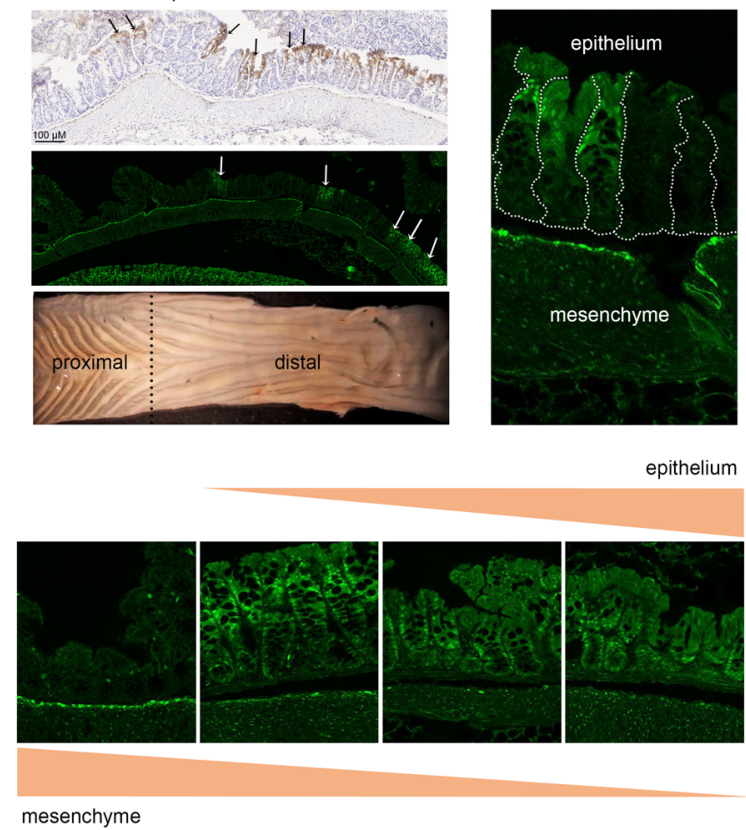

Fig. 2 Murine and human HOXA13 expression is subject to strict spatial control in the colon. a A representative example from 3 mice of a "Swiss roll" configuration of the large intestine of the Hoxa13-GFP heterozygous mouse model. An asterisk indicates the most distal portion of the epithelium. Magnification of the insets are shown in panels $\mathbf{b}, \mathbf{c}$ and $\mathbf{e}$. $\mathbf{b}$ The proximal border of physiological Hoxa13 expression in the adult mouse is patchy and located between the proximal and distal colon, indicated by a black dashed line in the bottom panel (macroscopic image of an opened mouse colon). Representative images of anti-GFP IHC and confocal microscopy are shown. Arrows indicate crypts that are positive for Hoxa13 among Hoxa13-negative crypts. c The Hoxa13 expression is crypt clonal. This is observed for $n=1$. d In adult humans the cecum bottom is negative for HOXA13 while positivity increases distally ( $n=5$ independent sampes). Mean \pm SEM, ${ }^{\star} p<0.05$, repeated measures ANOVA with Holm-Š́dák's multiple comparisons test, $p=$ 0.001. HOXA13 mRNA levels were normalized to levels in the transverse colon. e Hoxa13 expression is tightly regulated along the baso-luminal axis. Distally, Hoxa13 is expressed along the entire baso-luminal axis of the colonic crypts, proximally only expression at the luminal side is seen. In addition, a mesenchymal expression is observed in the cells just beneath the epithelium, predominantly in the proximal colon. Anti-GFP IHC and confocal images are shown.

esophagus during the epithelial transition period, reduced in adult squamous esophagus, and increase again in BE. Thus, the epithelium of both the human and mouse adult upper GI tract, in particular the GEJ and ESMGs for human, is characterized by the presence of a subpopulation of HOXA13/Hoxa13-positive cells in an otherwise HOXA13/Hoxa13 negative surrounding.

HOXA13 affects differentiation potential and posteriorizes. Having established that individual HOXA13-positive cells reside in the physiological upper GI tract and are enhanced in BE tissue, we next set out to investigate the potential role of this population of cells in the etiology of $\mathrm{BE}$. To this end, we further analyzed the single cell RNA-seq ${ }^{21}$ data set mentioned above. In this study, the GEJ was not sampled for analysis. However, the $8 \%$ of cells of the normal esophagus that express HOXA13 exhibit transcriptional overlap with cells derived from $\mathrm{BE}$ tissue as seen from the t-Distributed Stochastic Neighbour Embedding ( $t$-SNE) plot (Fig. 5a). Gene expression analysis indicates that these cells are derived from ESMG (Fig. 5b and Supplementary Data 1)21,23. Specifically, and in contrast to the HOXA13-negative cell population, $>70 \%$ of the HOXA13-positive cells from the normal squamous mucosa are positive for submucosal markers LEFTY1 and OLFM4, designated ESMG markers, which have also been described as markers of $\mathrm{BE}$ progenitor cells ${ }^{21}$. Additionally, HOXA13-positive cells express mucosal markers TFF3, Lyz and SOX9, as well as columnar and BE markers TFF1, KRT7, VIL1, MUC5B, MUC3A, MUC13, MUC1, and CEACAM5, while being negative for keratinization marker $I V L$ and basal epithelial cell marker $p 63$ (Fig. 5b and Supplementary Data 1 for the list of genes enriched in HOXA13-positive cells). In BE, the percentage of cells positive for these columnar and ductal markers increase also in the HOXA13-negative population, suggesting either that upon differentiation some of these cells might lose HOXA13 expression, or that there is more than one population giving rise to $\mathrm{BE}$ tissue. This would be in line with mouse data, as the murine esophagus lacks ESMGs and Hoxa13-positive cells. Interestingly, although rare in this dataset, within the $\mathrm{TFF}^{+}$population four cells were identified to be triple positive for KRT14 (a gene pair with KRT5), TP63 and KRT7 ${ }^{21}$ but these were not positive for HOXA13.

The cell of origin with respect to formation of the BE segment should be able to generate a variety of differentiated cell types that exhibit colonic, gastric, pancreatic acinar or other phenotypes ${ }^{24,25}$. In chick embryos, HOXA13 regulates regionalization after 1.5 days of development, showing the involvement of HOXA13 in early differentiation, consistent with an effect of this gene on cellular phenotype in such pluripotent progenitor cells $^{26}$. In an effort to experimentally test the influence of HOXA13 on cell fate, we generated HOXA13-inducible pluripotent mouse embryonic stem cells (mESCs). These pluripotent mESCs can be efficiently differentiated to multipotent definitive endoderm, as determined by membrane expression of CXCR4 and E-cadherin (Fig. 5c). This was further confirmed by RNAseq, showing a strong upregulation of definitive endoderm markers such as Sox17 and Foxal in these differentiated cells, while pluripotency markers such as Nanog are downregulated (see 


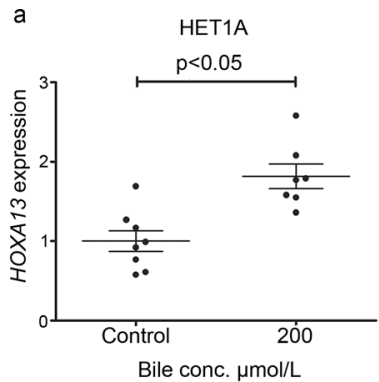

C

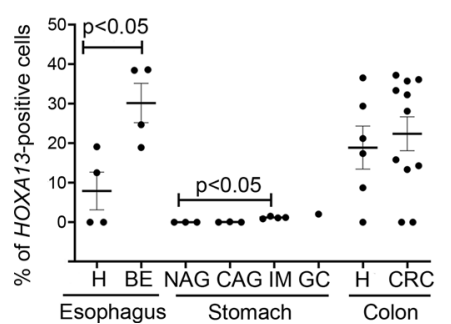

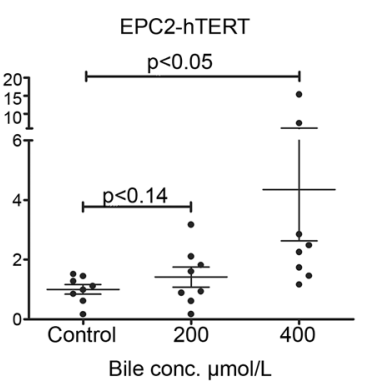

d
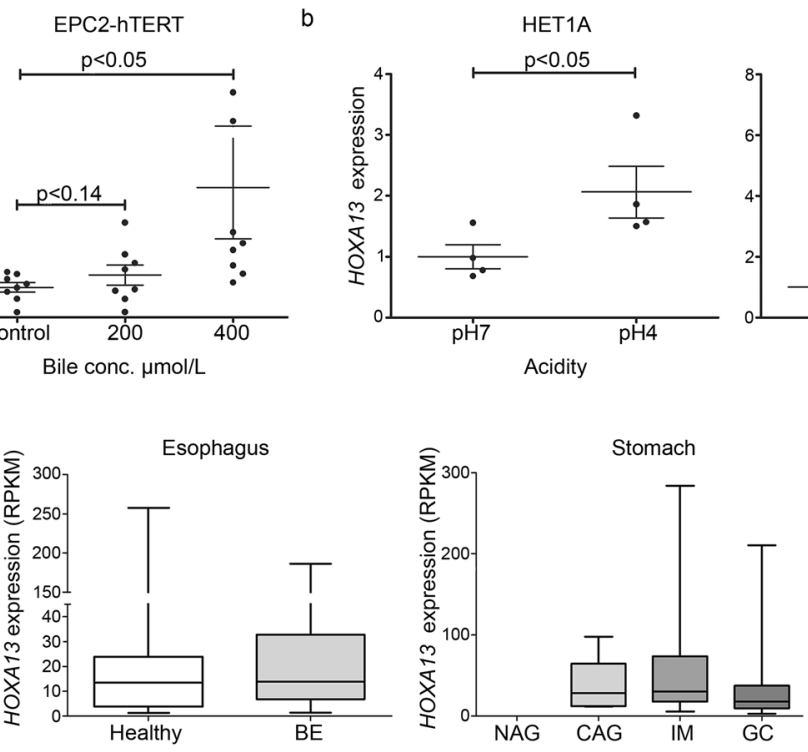

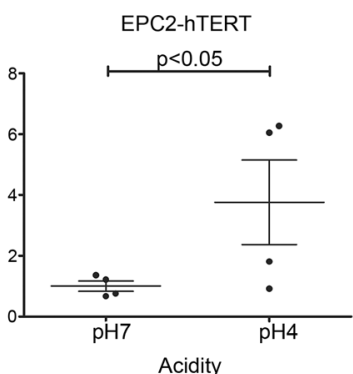

Fig. 3 Number of HOXA13 + cells rather than cellular expression levels are associated with metaplasia. Exposure to bile (at pH 7) (a) or to acid (b), in two in vitro model systems of gastroesophageal reflux disease (GERD), marginally induces the expression of $H O X A 13$ from low baseline expression levels in two primary immortalized squamous esophageal cell lines. Error bars represent the $95 \% \mathrm{Cl}$ of the mean. $n=4$ independent experiments. For Het1a, twotailed $t$-test was used ( $p=0.0096$ in a, $p=0.0322$ in b), for EPC2-hTERT Dunn's multiple comparisons test in a (control vs. $200 \mu M, p>0.99 ;$ control vs. $400 \mu \mathrm{M}, p=0.0112 ; 200 \mu \mathrm{M}$ vs. $400 \mu \mathrm{M}, p=0.14$ ), one-tailed $t$-test in $\mathbf{b}, p=0.0486$. c The number of HOXA13-postive cells are increased in Barrett's esophagus (BE) and intestinal metaplasia (IM) as compared to normal esophagus or stomach tissue. Healthy and BE esophageal samples are derived from the same patients. Mean \pm SEM are shown. $n=4$ individuals for esophagus, $n=3$ for NAG, CAG, $n=4$ for IM, $n=1$ for GC, $n=6$ for healthy colon, $n=11$ for CRC. Graphs are based on the analysis of single cell RNA data seq21, GSE13452082, GSE8186183. Two-tailed $t$-test was used for esophagus $(p=0.0443)$ and colon $(p=0.6808)$, one-way analysis of variance for stomach with Dunnett's Multiple Comparison Test ( $p<0.0001)$. d HOXA13 expression level per cell is unchanged. Expression level presented in $H O X A 13^{+}$cells only. $H$ healthy, $B E$ Barret's esophagus, $N A G$ non-atrophic gastritis, $C A G$ chronic atrophic gastritis, IM intestinal metaplasia of stomach, GC early gastric cancer, and CRC colorectal cancer. $n=37$ for $\mathrm{H}, n=132$ for $\mathrm{BE}$, NA for $\mathrm{NAG}$, $n=5$ for CAG, $n=163$ for IM, $n=69$ for GC, $n=37$ for healthy colon, $n=87$ for CRC of single cells from the individuals mentioned in (c). For GC, statistics are not presented as data per patient was not provided. Boxplots with middle line is the median, the lower and upper hinges correspond to 25 th to 75th percentiles, and whiskers representing min-max values.

Supplementary Table 1). Using ingenuity pathway analysis (IPA) to further analyze differently expressed genes, a positive association was found with "differentiation of embryonic cells" $\left(z=1.82, p=6.38 \times 10^{-15}\right)$. Intriguingly, when HOXA13 expression was induced, cells differentiated less effectively towards definitive endoderm as determined by $\mathrm{CXCR} 4^{+} / \mathrm{E}^{- \text {cadherin }^{+}}$ expression and morphological assessment (Fig. 5c, d). Consistent with a reduced unilinear differentiation, clones expressing HOXA13 showed greater expansion (Fig. 5d).

We next contrasted the transcriptome of non-differentiated, pluripotent HOXA13-overexpressing and control cultures to identify potential molecular mediators of the HOXA13 effects observed. Results of IPA analysis of differential gene expression are broadly consistent with HOXA13 conferring a pluripotent phenotype. Specifically, forced HOXA13 expression results in upregulation of the "role of Nanog in mammalian embryonic cell pluripotency" category $\left(z=1.34, p=2.32 \times 10^{-3}\right)$, an effect that involves Sox2, Nanog, Tbx3, Hesx-1, and Dppa-1 amongst others $^{27,28}$ (See Table 1 for more details/results, fold changes, and $q$-values with regard to this experiment). HOXA13 expression also appears to downregulate Wnt signaling, possibly through BMP signaling ${ }^{29}$. Wnt signaling is known to promote mesoendodermal differentiation ${ }^{30}$, these results are consistent with HOXA13-mediated downregulation of Wnt signaling during axial elongation $^{31}$. Thus, the transcriptional profile provoked by HOXA13 is consistent with maintaining a relatively pluripotent phenotype which in turn may increase compartment expansion.

HOXA13 expression does not block endodermal differentiation of mESC cells completely, suggesting that a role for HOXA13 in this compartment is still relevant. Definitive endoderm is a feature of the entire GI tract epithelium, and does not distinguish upper and lower GI epithelium per se. To investigate the role of HOXA13 in this cell compartment and test our prediction that HOXA13 expression would predispose endoderm to acquire distal phenotypes, we sorted $\mathrm{CXCR} 4^{+} /$E-cadherin $^{+}$cells of $\mathrm{HOXA13}$ positive and negative cultures and contrasted their mRNA expression. HOXA13 upregulates gene expression associated with determination of morphology in definitive endoderm cells. In IPA analysis, "actin cytoskeleton signaling" was most activated $(z=$ $\left.3.00, p=3.74 \times 10^{-2}\right)$. "RhoA signaling", which stimulates actin polymerization, $\left(z=2.12, p=1.12 \times 10^{-2}\right)$ was also stimulated. HOXA13 supports distal epithelial functions with upregulation of microvillus-associated genes, Ezr and Vill, keratins, Krt19 and $K r t 20$, tetraspan network genes, Igsf8, and exocrine function associated genes such as Gcnt3, normally expressed in the distal GI-tract epithelium ${ }^{32}$. In addition, more transcripts of "Cell proliferation of carcinoma cell line" $\left(z=1.13, p=1.5 \times 10^{-6}\right)$ and "Neoplasia of cancer cells" $\left(z=1.13, p=2.22 \times 10^{-4}\right)$ categories, such as Fgfr2 and Nek2, were detected. Thus, forced HOXA13 expression during endodermal differentiation supports caudal epithelial functions and proliferative potential (see Table 1 for fold changes and $q$-values; see Supplementary Data 2 for additional relevant molecules).

Together, these data are in apparent agreement with HOXA13expressing cells displaying a progenitor phenotype and having a competitive advantage, while simultaneously driving the acquisition of a more distal columnar phenotype once committed to differentiation (see Fig. 5e). 

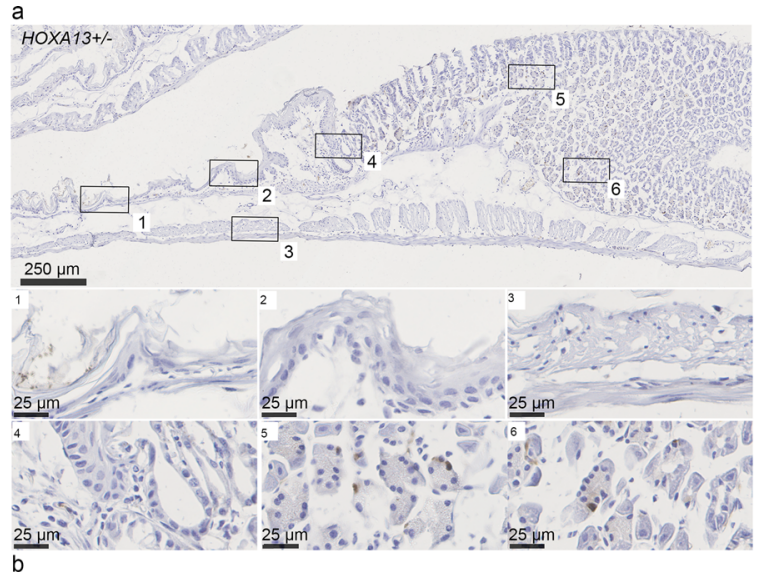
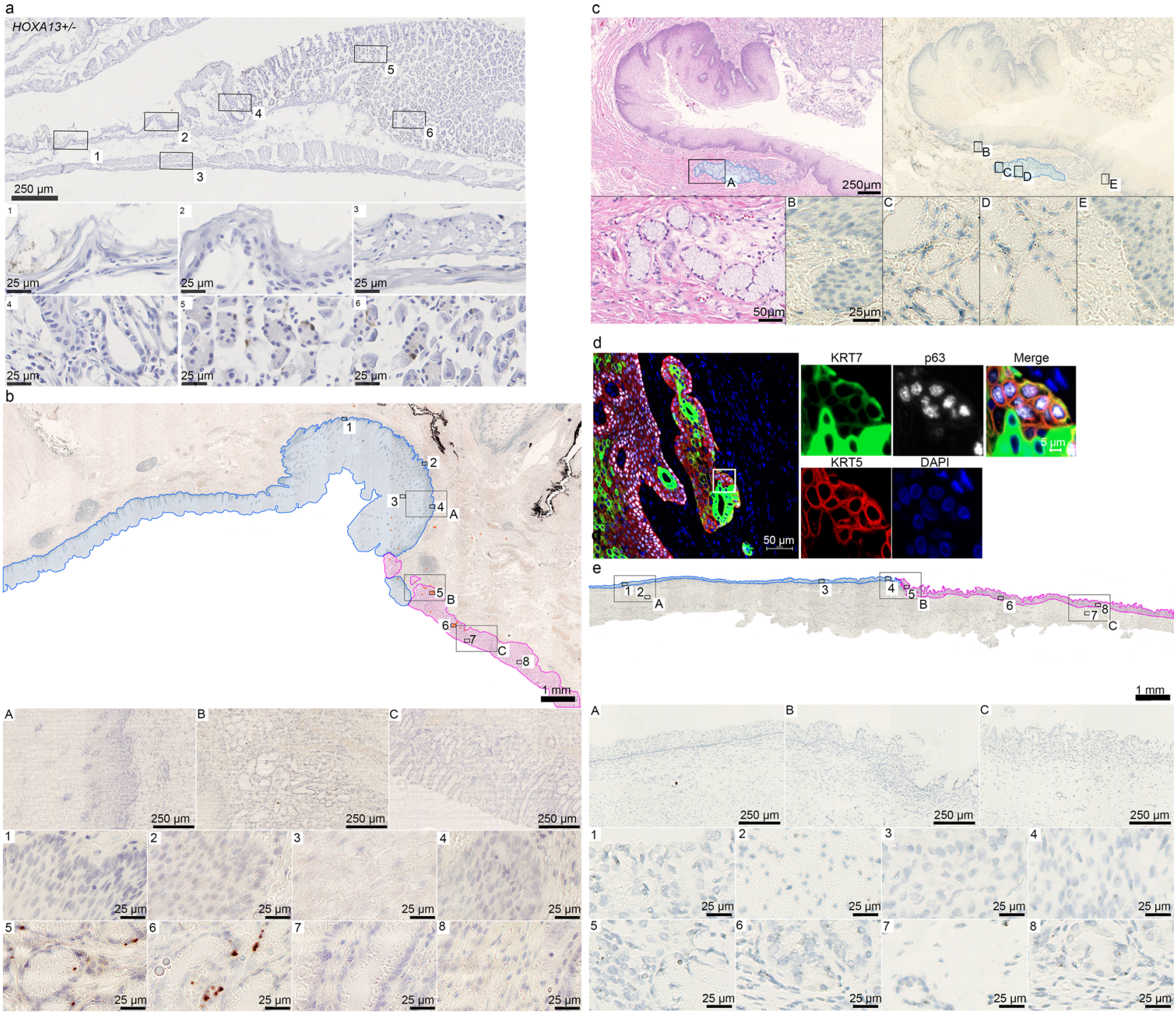

Fig. 4 HOXA13 expression in the upper GI tract. a Representative example of anti-GFP immunohistochemistry of a Hoxa13-GFP heterozygous mouse with gastroesophageal junctions (GEJ) (SQ; squamous epithelium, ST; stomach) ( $n=3$ ). Hoxa13 is expressed in single cells of the stomach starting from the GEJ (5-6) and absent in the esophagus and stroma (1-4). b HOXA13 expression as measured by RNA ISH in a representative example from $n=3$ with similar results of an adult human GEJ with magnification panel of: A-esophagus, B-GEJ area, C-proximal stomach. Orange circles indicate the positive signal in the overview image. c HOXA13 expression as measured by RNA ISH in human esophageal submucosal gland (ESMG) with magnification panel of: $A-H \& E, C, D-E S M G, B, E-$ squamous esophagus, $n=1$. d Keratin 7 (KRT7), keratin 5 (KRT5) and p63 triple positive cells are found in the ESMG, $n=1$. e Overview of a representative example of a 17-week old fetus GEJ: A) Stratified esophageal epithelium of the distal esophagus (blue), B) GEJ area, C) gastric epithelium of the proximal stomach (pink), $n=3$.

HOXA13 and the chromosome 1 epidermal differentiation complex. Further support for a role of HOXA13 in the loss of the squamous phenotype and the appearance of caudal columnar phenotypes in the esophagus comes from experiments in which we investigated the effect of HOXA13 directly on esophageal cell models. To this end, we used CRISPR-Cas9 technology to delete HOXA13 from BAR-T, a primary monoclonal immortalized cell line derived from metaplastic tissue of a BE patient, with cells expressing both columnar and squamous markers ${ }^{33}$. Three separate HOXA13 knock-out clones were selected to circumvent potential off target effects. Reversely, we provoked lentivirusmediated HOXA13 expression in EPC2-hTERT, an immortalized squamous esophageal cell line. For these latter experiments we used a mixed cell population of lentivirally transduced cells as to avoid clonal artifacts influencing results. Transcriptomes in these two models (Fig. 6a) were contrasted to their respective control lines.
There was substantial overlap in the gene sets significantly affected by losing HOXA13 in BAR-T compared with those significantly affected by gaining HOXA13 in EPC2-hTERT, taking into account the direction of regulation $\left(\mathrm{X}^{2}\right.$ test: $p=4.74 \times 10^{-34}$ ) (see Supplementary Data 3 ). Investigation of this overlap across the two technically independently generated datasets limits the incidence of chance findings or single model system bias. Overlapping genes positively affected by HOXA13 expression in esophageal cells are IL7r, FAM196B, ADAMTS6, NRG1, LTBP1, JAG1, ELL2, SMAD7, C12ORF75, AXL, TIPARP, IKBIP, DUSP7, and GOLIM4. Downregulated by HOXA13 expression are SERPINB13, MYO5C, KLK7, ANXA9, TMPRSS4, TTC9, MATN2, TNFAIP2, RAB27B, HCAR2, C6ORF132, EXPH5, MAP3K5, and FUCA1. IPA analysis of the results predicts an increase in "(malignant) cell transformation" $\left(z=2.00, p=5.81 \times 10^{-3}\right)$ and a decrease in "inflammation of an organ" $\left(z=-2.59, p=8.29 \times 10^{-3}\right.$; gene function is described in 
a

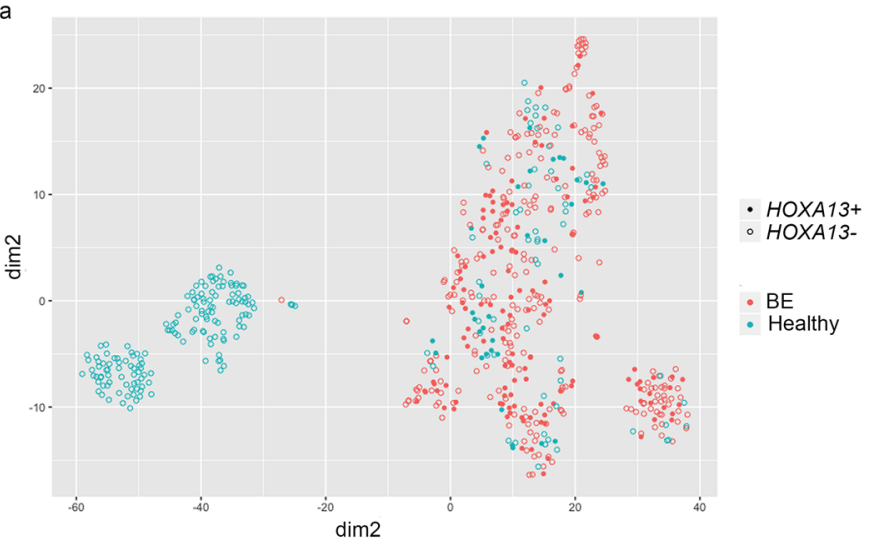

b

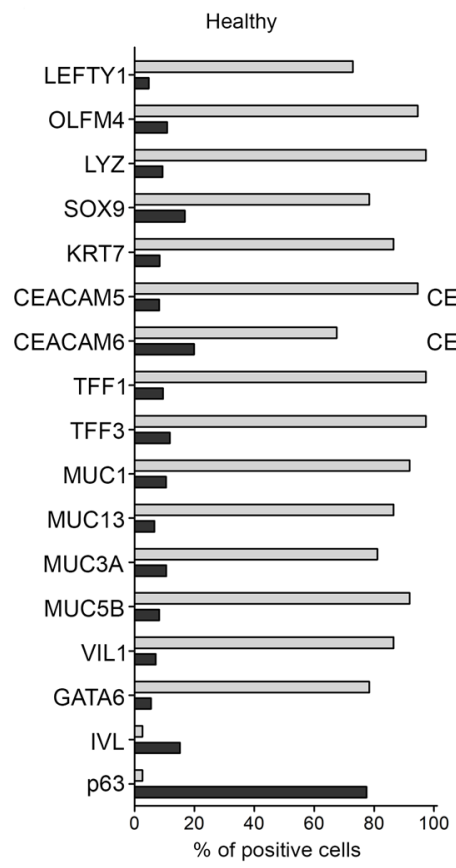

BE

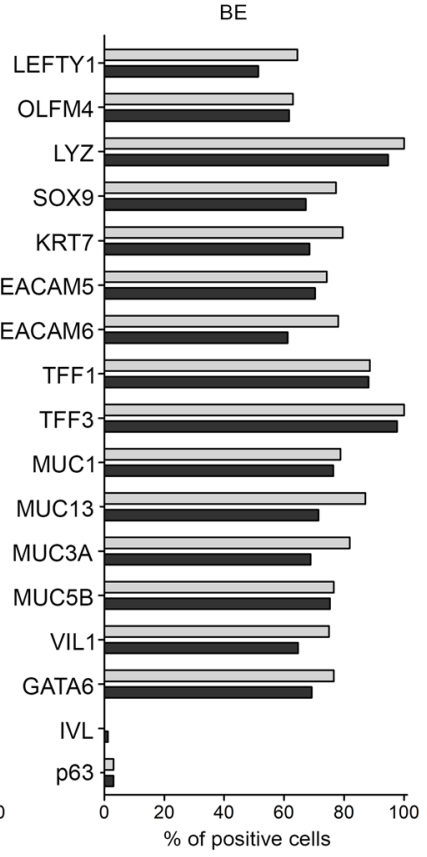

d

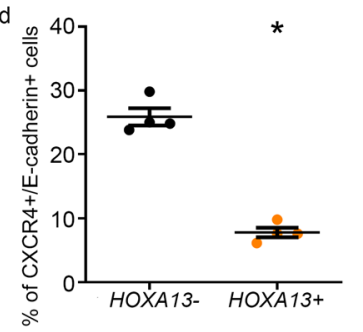

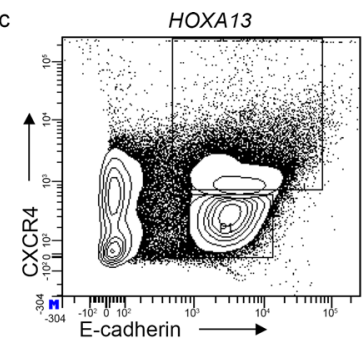
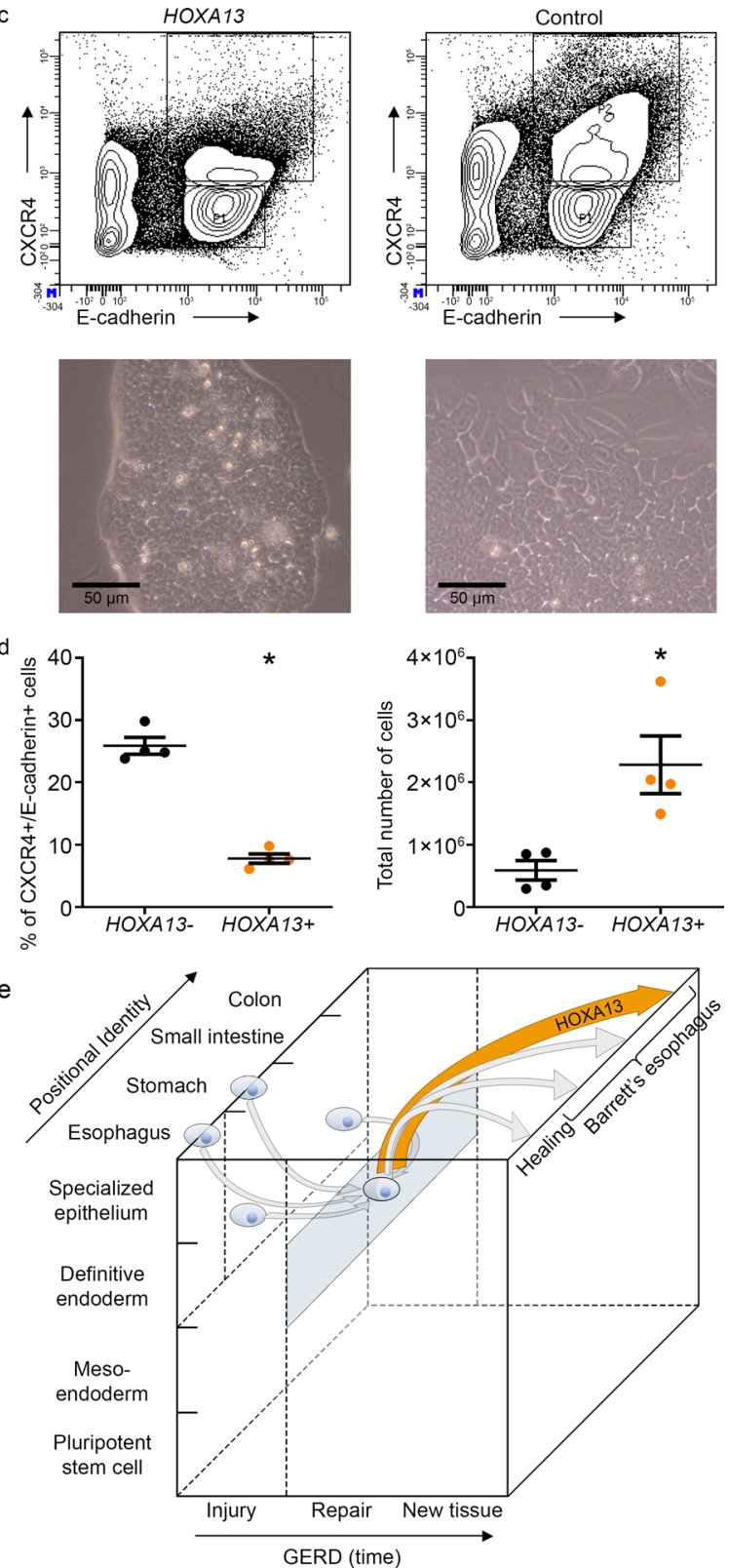

Fig. 5 HOXA13 cellular expression modulates cell fate. a HOXA13+ cells of normal esophagus cluster together with BE cells in t-distributed Stochastic Neighbor Embedding ( $T$-SNE) plot based on single cell RNA expression profiling ${ }^{21}$. b Analysis of single cell RNA seq data revealed that in contrast to HOXA13- cells, HOXA13+ cells express submucosal gland markers, Barrett's esophagus (BE) markers and have decreased expression of squamous markers (p63, IVL) in healthy esophagus. This difference is not observed in BE. $n=846$ of HOXA13- cells in healthy esophagus, $n=37$ of HOXA13 ${ }^{+}$in healthy esophagus, $n=263 \mathrm{HOXA13}^{-}$cells in $\mathrm{BE}, n=132$ of HOXA13+ cells in BE. $\mathbf{c}$ HOXA13-overexpressing definitive endoderm is relatively resistant to terminal differentiation. Mouse embryonic stem cells (mESC) cells with and without forced HOXA13 expression were differentiated from pluripotent stem cells to definitive endoderm. The percentage of differentiated definitive endoderm cells, defined as CXCR4 ${ }^{+} / \mathrm{E}$-cadherin ${ }^{+}$cells, was analyzed by FACS analysis (upper panels). Lower panels (representative light microscopy images) show morphological differences in cultures of HOXA13 overexpressing and wildtype mESCs upon differentiation to definitive endoderm, which induces a flattening of cell layers, with larger and irregular shaped cells. d Quantification of FACS analysis results indicates that the percentage of $\mathrm{CXCR} 4^{+} / \mathrm{E}$-cadherin ${ }^{+}$cells is decreased in HOXA13-overexpressing cell cultures under differentiation conditions ( $p$ 0.0001). HOXA13-expressing cells expand faster during the differentiation process compared to control cells (total number of cells increased) $(p=0.0135)$. Mean \pm SEM, ${ }^{\star \star \star} p<0.001, n=4$ independent experiments, $t$-test (two-tailed). e Model of cellular identity in BE development. The $X$-axis represents time (hypothetical units) following exposure to GERD-inducing agents. $Y$-axis shows differentiation during embryology and pathology. $Z$-axis indicates the positional identity of GI-tract tissues. Several theories exist regarding the cell of origin of BE: they may be fully differentiated esophageal or stomach cells, or less differentiated cells within these organs (depicted by the 4 cells on the $Y-Z$ plane). Irrespective of its location or differentiation state, this cell or origin might lose its correct positional identity or maintain its aberrant positional identity and resembles a definitive endoderm like cell. This is visualized by the blue rectangle harboring the cell with the thicker blue contour. For the model of cellular identity in BE, our data suggest that HOXA13 expressing clones in the GEJ, depicted in orange, may outcompete clones with another positional identity, providing an explanation for the distal phenotype observed in $\mathrm{BE}$. 


\section{Table 1 Fold changes and q-values for the mRNAs mentioned in the results section of the main text pertaining to cell culture models analyzed by RNA-Seq. \\ Gene name Fold change $q$-value (multiple testing corrected $p$ value)}

Forced HOXA13 in mESC confers a relative competitive advantage in multipotent cell cultures through upregulation of Nanog signaling and downregulation of Wnt signaling

$\begin{array}{lll}\text { Sox2 } & 1.43 & 0.01 \\ \text { Nanog } & 1.47 & 0.00 \\ \text { Tbx3 } & 3.53 & 0.00 \\ \text { Hesx-1 } & 20.56 & 0.00 \\ \text { Dppa-1 } & 64.95 & 0.00 \\ \text { Igf2 } & 3.61 & 0.00 \\ \text { Wnt3 } & 0.43 & 0.00 \\ \text { Wnt4 } & 0.36 & 0.00 \\ \text { Wnt6 } & 0.36 & 0.00 \\ \text { Wnt8a } & 0.48 & 0.01 \\ \text { Sp8 } & 0.12 & 0.00 \\ \text { Lef1 } & 0.34 & 0.00 \\ \text { Tbxt } & 0.41 & 0.00 \\ \text { Axin2 } & 0.55 & 0.04 \\ \text { Fgf8 } & 0.10 & 0.00 \\ \text { Cdx1 } & 2.47 & 0.00 \\ \text { Grhl3 } & 5.00 & 0.00 \\ \text { Vill } & 1.87 & 0.00\end{array}$

Forced HOXA13 expression supports caudal epithelial functions and appears to promote proliferation in $\mathrm{DE}$

Sox17 $\quad 12.55 \quad 0.00$

$\begin{array}{lll}\text { Lgr5 } & 5.39 & 0.00\end{array}$

$\begin{array}{lll}\text { Nanog } & 0.20 \quad 0.02\end{array}$

$\begin{array}{lll}\text { Ezr } & 2.20 & 0.00\end{array}$

$\begin{array}{lll}\text { Vill } & 2.58 & 0.048\end{array}$

$\begin{array}{lll}\text { Krt19 } & 2.34 & 0.00 \\ \text { Krt } & \end{array}$

$\begin{array}{lll}K r t 20 & 2.84 & 0.01\end{array}$

$\begin{array}{lll}\text { lgsf8 } & 4.67 & 0.00\end{array}$

Gent3 $\quad 3.51 \quad 0.00$

$\begin{array}{lll}\text { Fgfr2 } & 2.81 & 0.00\end{array}$

Nek2 $\quad 2.35 \quad 0.01$

HOXA13 downregulates the chromosome 1 epidermal differentiation complex, is pro-oncogenic, and conveys typical characteristics of the $\mathrm{BE}$ phenotype

$\begin{array}{lll}\text { ANXA9 } & 0.48 & 0.04\end{array}$

$\begin{array}{lll}\text { EVPL } & 0.61 & 0.03\end{array}$

$\begin{array}{lll}\text { SCEL } & 0.52 & 0.01\end{array}$

$\begin{array}{lll}K L K 7 & 0.42 & 0.01\end{array}$

$\begin{array}{lll}\text { EMP1 } & 0.56 & 0.03\end{array}$

$\begin{array}{lll}\text { SERPINB13 } & 0.38 & 0.00\end{array}$

$\begin{array}{lll}\text { DLL1 } & 2.57 & 0.00\end{array}$

$\begin{array}{lll}\text { FURIN } & 1.49 & 0.03\end{array}$

$\begin{array}{lll}J A G 1 & 1.85 & 0.04\end{array}$

Supplementary Data 3) in cells expressing HOXA13. Intriguingly, HOXA13 downregulates the epidermal differentiation complex (EDC); Fig. 6b, c). The EDC, located on chromosome 1q21.3, contains clustered multigene families of genes associated with cornified envelope formation in stratified squamous epithelia, such as the S100 and the small proline-rich region (SPRR) genes ${ }^{34}$. Among the overlapping downregulated genes in both cell models, ANXA9 is also associated with differentiating keratinocytes ${ }^{35}$, and $E V P L, S C E L$, and KLK7 are cornified envelope genes ${ }^{36-38}$. EMP1 and SERPINB13 downregulation is associated with increased disease severity in gastric cancer $(E M P 1)$ and head and neck squamous cell carcinoma (SCC) (SERPINB13) ${ }^{39,40}$. See Table 1 for fold changes and $q$-values and Supplementary Data 3 for more differentially expressed molecules related to morphology. The downregulation of a gene region known to be essential for maintaining a squamous phenotype provides mechanistic support to the notion that altered HOXA13 expression is cardinal for provoking the BE phenotype.

These experiments also provide mechanistic support for the notion that HOXA13 expression may offer an explanation as to why $\mathrm{BE}$ is prone to progression to EAC. HOXA13 mediates down-regulation of the EDC and many EDC and cornified envelope genes are progressively down-regulated in the $\mathrm{BE}$ to EAC cascade ${ }^{36,41}$. In BE, EAC, and esophageal SCC, loss of heterozygosity $(\mathrm{LOH})$ of the EDC is common ${ }^{42-44}$. Low EDC gene expression predicts chemotherapy non-response and $\mathrm{LOH}$ of the EDC is associated with reduced survival in curatively treated EAC patients ${ }^{43,45}$. In our experimental models, we observed HOXA13-mediated upregulation of genes associated with Notch signaling, specifically DLL1, FURIN, and JAG1. Notch signaling is associated with malignant transformation ${ }^{46,47}$. In IPA analysis "Non-melanoma solid tumor" $\left(z=2.03, p=9.28 \times 10^{-8}\right)$ and "invasion of cells" $\left(z=2.08, p=2.47 \times 10^{-3}\right)$ were shown to be activated by HOXA13, whereas HOXA13 expression negatively influenced the "Apoptosis" $\left(z=-1.52, p=2.23 \times 10^{-3}\right)$ and "killing of cells" $\left(z=-2.03, p=2.03 \times 10^{-3}\right)$ categories. Many individual genes showed differential regulation in a prooncogenic direction (see Supplementary Data 3).

In conclusion, using HOXA13 knock-out and overexpression in a Barrett's and a squamous cell line, we show that HOXA13 downregulates the epithelial differentiation complex and other cornified envelope genes which normally function to maintain squamous epithelial morphology and act as tumor suppressor genes. Additionally, Notch signaling is overexpressed and many individual genes show differential regulation in a pro-oncogenic direction.

HOXA13 supports columnar phenotype and provides proliferative advantage. Having established the transcriptional effect of HOXA13 on BAR-T and EPC2-hTERT cells, we next investigated the functional consequences of HOXA13 in these cells. As was seen for mESCs, HOXA13 expression significantly enhances the growth-rate of esophageal cells. For BAR-T cells, a proliferative advantage of HOXA13 expression was seen in $2 \mathrm{D}$ cultures (Fig. 6d), while for EPC2-hTERT the positive effect of HOXA13 expression on cell growth was more noticeable under $3 \mathrm{D}$ culture conditions (Fig. 6e). Moreover, HOXA13 expression decreases the sensitivity of keratinocytes to bile/acid exposure (Fig. 6f), consistent with the notion that HOXA13 confers cellular protection under GERD-like conditions.

To gain further insight into the role of HOXA13 in cell morphology and organization, we made use of the fact that EPC2-hTERT cells can be differentiated in 3D spheroid cultures, and become organized in layers with a more flattened cytological aspect in the middle of spheroids and high expression of keratinization markers such as involucrin, similar to esophageal stratified epithelium (see example in Fig. 7a top panel for differentiated morphology $)^{48}$. Upon overexpression of HOXA13, EPC2-hTERT spheroids increase in size while maintaining a less differentiated phenotype (undifferentiated morphology, Fig. 7a bottom panel). Quantification of these morphological states indicates that in control cultures, $80 \%$ of spheroids attain a stratified epithelial phenotype, while overexpression of HOXA13 reduces this number to $28.6 \%$ ( $p<0.05$, Fig. $7 \mathrm{~b}$ left panel). This was further confirmed by staining for involucrin as a marker of keratinization, showing a decreased expression in spheroids derived from HOXA13-overexpressing EPC-hTERT cells $(p<0.05$, Fig. $7 \mathrm{~b}$ right panel). Thus, in primary immortalized esophageal cells HOXA13 overexpression reduces keratinization. 

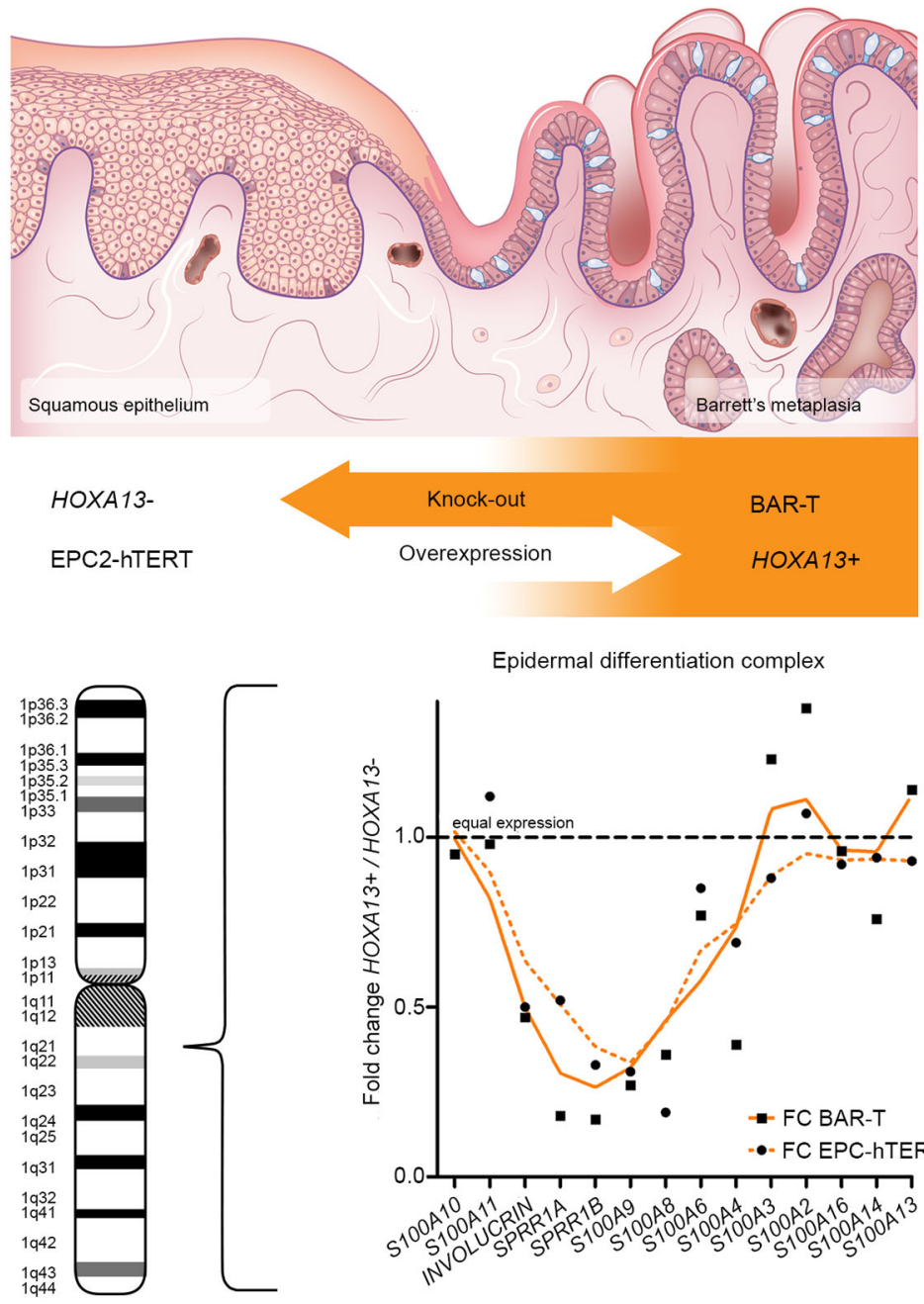

Epidermal differentiation complex

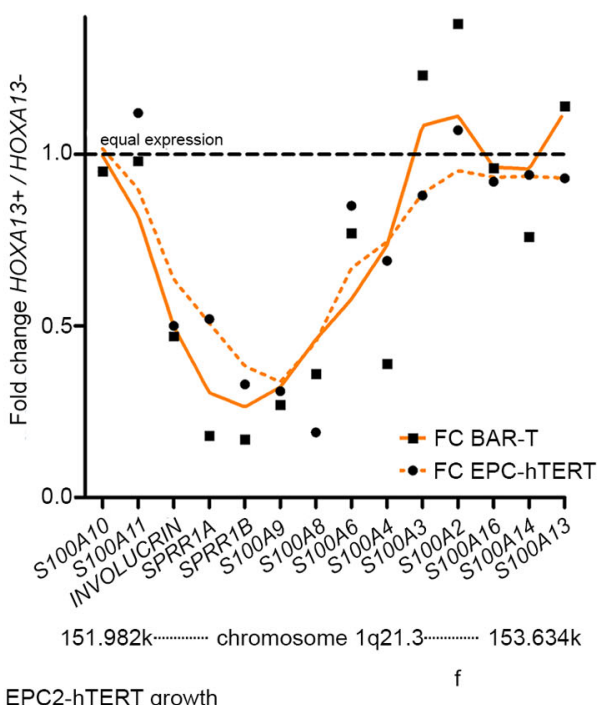

d
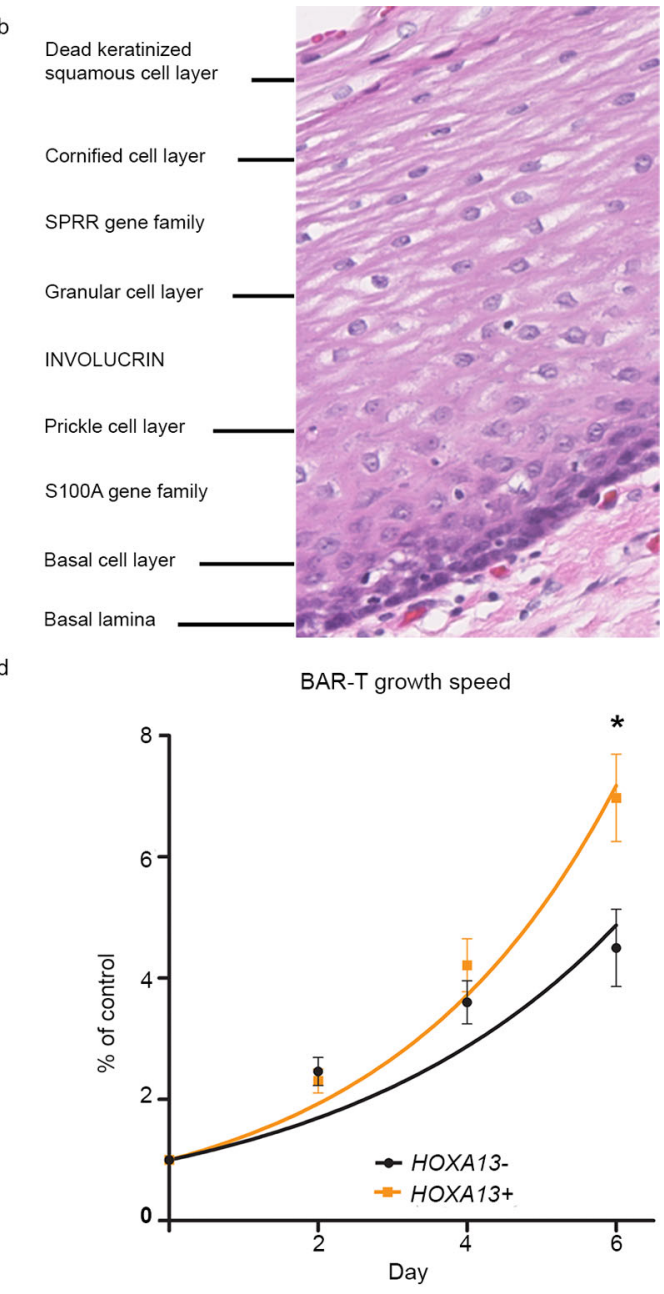

Bile/acid treatment of EPC2-hTERT cells

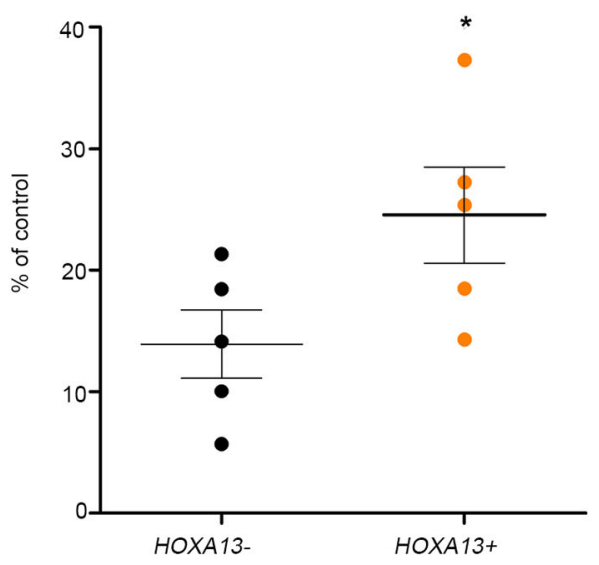

We further investigated the morphological role of HOXA13 in the BE-derived BAR-T cell line. In $2 \mathrm{D}$ cultures, an altered spatial distribution in growth pattern was observed, with cells growing more closely together in the absence of HOXA13 suggesting an effect on tissue morphology (Fig. 7c). The BAR-T cell model also allows testing the effect of HOXA13 on columnar versus squamous differentiation in in vitro and in vivo settings. A $3 \mathrm{D}$ in vivo tissue reconstitution model was employed in which BAR$\mathrm{T}$ cells were grafted in the lumina of devitalized and denuded rat tracheas and implanted in NOD SCID mice. Under these conditions, parental non-transfected BAR-T cells produce both intestinal-type columnar epithelium and stratified squamous epithelium from the same clone. Hence this cell line has the potential to produce two types of morphological distinct epithelia $^{33,49}$ (Fig. 7e and Supplementary Fig. 9). Thus the epithelium in the model finds itself on a tipping point between both morphologies. This characteristic makes the in vivo tissue reconstitution model suited for studying the influence of 
Fig. 6 HOXA13 counteracts squamous identity and increases growth of esophageal cells. a Two models were constructed to investigate the function of HOXA13 at the gastroesophageal junctions (GEJ). One model used EPC2-hTERT, a primary immortalized human squamous esophageal cell line, characterized by low HOXA13 expression, in which HOXA13 was transduced. The second model employed BAR-T, a primary immortalized human Barrett's esophagus (BE) cell line, characterized by high HOXA13 expression, in which HOXA13 was knocked out. b Hematoxylin and eosin (H\&E) staining of the squamous esophagus of a patient without BE indicating the expected location of some of the products of the Ch1q21.3 epidermal differentiation complex along with other genes from the cornified envelope of the epidermis. c HOXA13 leads to a downregulation of genes in the Ch1q21.3 epidermal differentiation complex in both model systems. A cubic spline fit of HOXA13 mRNA regulation is shown, with the BAR-T control transduced cell line presented compared to its HOXA13 knock-out counterpart, and HOXA13 overexpressing EPC2-hTERT cells presented compared to their parental line. FC fold change. d HOXA13 knock-out in a BE cell line reduces the growth of the cell pool, as measured by MTT assay. Mean \pm SEM, ${ }^{*} p<0.05$, exact $p=0.0204$, two-tailed $t$-test, $n=$ 9 independent experiments. e HOXA13 overexpression in a EPC2-hTERT cell line increases its growth in 3D culture (area of spheroids, mean \pm SEM, ${ }^{*} p<$ $0.05, p=0.0174$, two-way ANOVA with Sidak's multiple comparisons test). f EPC2-hTERT cells with HOXA13 overexpression are less sensitive to bile/ acid exposure $(p=0.0343)$. MTT data presented as $\%$ of corresponding vehicle-treated controls. Mean \pm SEM, ${ }^{*} p<0.05, t$-test (two-tailed).

modulators of morphology, i.e. to show if the modulator favours intestinal-type columnar epithelium or stratified squamous epithelium. Studying the effect of HOXA13 knock-out, two important observations were made. Firstly, HOXA13 knock-out decreases the length of columnar-like epithelium which contains PAS positive cells and is negative for involucrin (Fig. 7e, f). Thus, loss of HOXA13 counteracts the proliferation of the intestinaltype columnar epithelium while the stratified squamous epithelial proliferation remains present. Secondly, HOXA13 knock-out impairs epithelial proliferation in general, as inferred from the thickness of the epithelial layer (Fig. 7d; Supplementary Fig. 9b). In vitro $2 \mathrm{D}$ organotypic ALI cultures of these cell lines confirm the in vivo findings, with $H O X A 13$ knockout reprogramming the BAR-T epithelial cells towards a squamous keratinized differentiated epithelium (Fig. 7f). In conclusion, HOXA13 supports intestinal-type columnar epithelial differentiation and proliferation of the Barrett's epithelium confirming the notion that HOXA13 expression can mediate both a competitive advantage as well as a predisposition to the formation of columnar phenotypes.

\section{Discussion}

In this study, we characterized $H O X$ gene expression and localization in mice and men, demonstrating a collinearity of these genes along the GI tract. Following analysis of one of these $H O X$ genes, HOXA13, we observed single HOXA13 + cells in the upper GI tract, which present exceptions to the $H O X$ gene collinearity theory. Specifically, in the normal physiology of the esophagus and proximal stomach, non-squamous structures such as the epithelium at the GEJ, glandular cells of ESMGs and glands of stomach contain single cells expressing HOXA13. The fact that these cells have not been described before may be a reflection of the fact that homogenization of tissues for qPCR masks this fraction, and that single cell analysis of the GI tract for this gene has not been performed before. We observe that GI pathology with distal phenotypes like intestinal metaplasia of the esophagus and stomach are characterized by an expansion of HOXA13positive cells, while conversely, a relatively low expression of HOXA13 is found in the phenotypically rostral Paneth cell metaplasia and pyloric metaplasia of the colon, compared to the surrounding physiological tissue.

It is clear that in normal physiology, HOXA13 contributes to the distal phenotype of the caudal GI tract, begging the question as to the role and origin of the HOXA13-expressing compartment now observed in the upper GI tract. We demonstrate that esophageal HOXA13-positive cells express columnar and BE markers and show gene expression patterns overlapping with $B E$-derived cells. Functionally, HOXA13 provides cells with several properties required for development of a BE segment. HOXA13 maintains cells in a stem-like progenitor state, while conferring a proliferative advantage, promoting cellular migration ${ }^{50}$ and resistance to bile and acid exposure. Furthermore, in cells that are lineage committed, HOXA13 supports a phenotypically columnar phenotype, most likely partly driven by downregulation of the chromosome 1 epidermal differentiation complex. Thus, our data are consistent with the hypothesis that $\mathrm{BE}$ arises as a consequence of the expansion of resident HOXA13-positive cells under abrasive environments such as GERD. Several potential theories have been proposed as to the origin of BE: transdifferentiation of basal cells in the squamous epithelium, extension of a special population of cells from the GEJ, repopulation of the esophagus after injury with cells derived from progenitors ESMGs or ducts, resident embryonic stem cells or circulating bone marrow cells ${ }^{51}$. These potential sources of esophageal columnar epithelium are not mutually exclusive, and BE may have more than one precursor cell or location. Our study supports the previously proposed hypothesis that BE may originate from ESMGs and the GEJ as HOXA13 is expressed in OLMF4 ${ }^{+}$, LEFTY $^{+}{ }^{+}$cells of ESMGs, recently suggested as a cell of $\mathrm{BE}$ origin ${ }^{21}$. The fact that in addition to the GEJ, rare HOXA13+ cells are found in the human esophagus and stomach, is consistent with the observation that after esophagogastrostomy $\mathrm{BE}$ can reoccur in patients, indicating that the involvement of the GEJ is not an absolute prerequisite for the development of $\mathrm{BE}^{52}$. Furthermore, our data show that HOXA13 is already present at stem cell level, supportive of the notion that BE may arise from a cell with stem-cell like characteristics. While HOXA13 expression overlaps greatly with KRT7, a columnar cytokeratin seen in Barrett's, we did not observe direct transcriptional overlap with the previously described $K R T 7^{+} K R T 14 / 5^{+} T P 63^{+}$cell of BE origin. However, $\mathrm{KRT7}^{+} \mathrm{KR}^{+} \mathrm{TP} 63^{+}$cells gave rise to BE-like epithelium only upon ectopic expression of $C D X 2^{22}$. Lineage-tracing studies are needed to further confirm whether one or more types of cells of origin might exist for BE. While we focused on HOXA13 here, it is conceivable that other $H O X$ paralogues are involved in $\mathrm{BE}$ pathophysiology, in particular caudal genes such as $\operatorname{HOXA10,11,}$ $B 13$, and $C 10$ are interesting candidates for further investigation, in particular as disruption of collinearity was reported for cluster $\mathrm{B}$ in $\mathrm{BE}^{9}$ and in duodenum of murine embryos ${ }^{12}$.

$\mathrm{BE}$ is considered as the precursor lesion for EAC, a dangerous form of cancer of which the incidence has substantially increased in recent decades. Increased insight into the pathogenesis of $\mathrm{BE}$ may aid development of prevention and treatment strategies for EAC. HOXA13 is involved in $\mathrm{ESCC}^{53}$ and other types of cancer ${ }^{54-57}$. Here we show that expression of HOXA13 also increases in EAC and colorectal cancer, provides proliferative advantage to the cells and activates cancer-related gene transcription like Notch signaling. Hence, we speculate that HOXA13 may play a role in BE progression towards EAC.

In toto, the present study identifies a importance of regional patterning by HOX genes in the gut epithelium. In Barrett's esophagus, gastric IM, and heterotopia of the upper GI-tract, a colon-like HOX gene expression is present, especially 
a

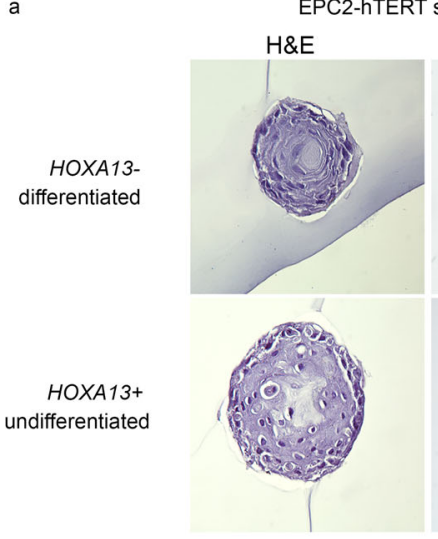

b

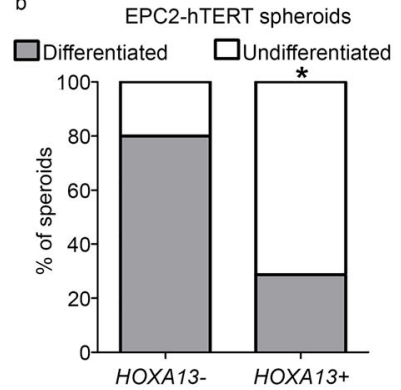

d

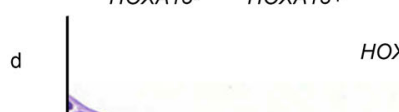

OXA13-
IVL

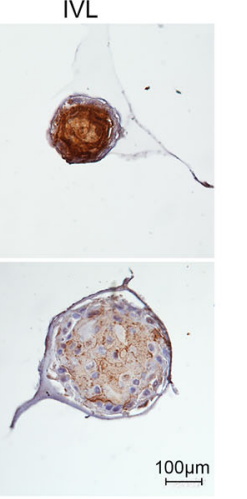

EPC2-hTERT spheroids

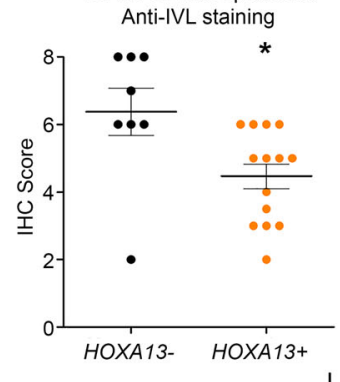

c

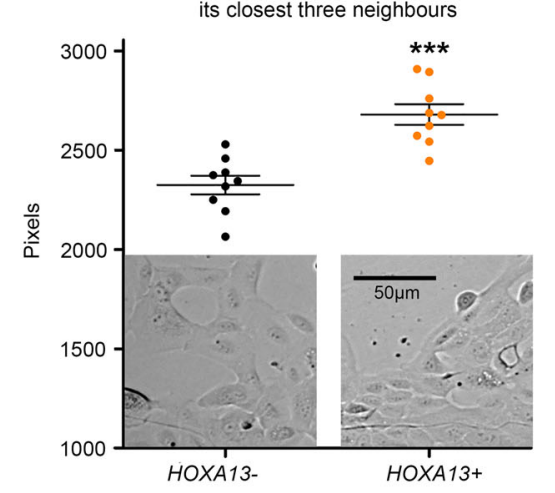

e

BAR-T cells, the length of
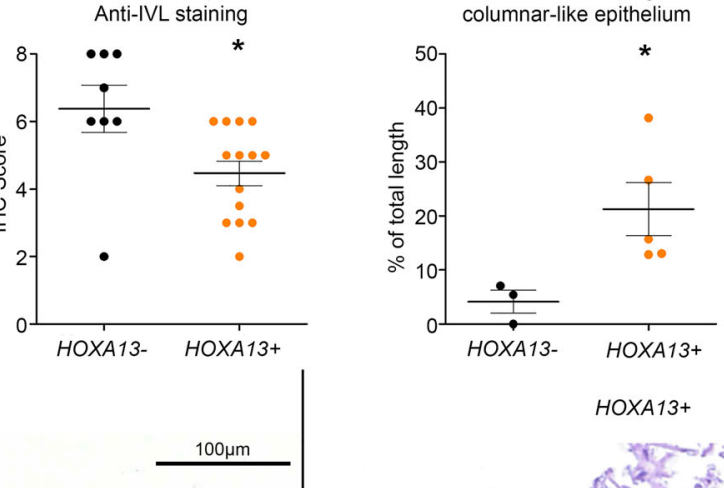
Fig. 7 HOXA13 supports intestinal-type columnar epithelial differentiation. HOXA13 overexpression impairs squamous differentiation of EPC2-hTERT spheroids as seen from the representative pictures (a) and quantitative assessment of morphologies based on hematoxylin and eosin (H\&E) or antiinvolucrin (IVL) immunohistochemistry $(\mathrm{IHC})(p=0.0086)$. (b). Median with interquartile range, ${ }^{*} p<0.05$, Mann-Whitney test (two-tailed). c HOXA13 knockdown (KO) affects spatial distribution of BAR-T cells. Mean \pm SEM, ${ }^{\star \star \star} p<0.001, p=0.0001, t$-test (two-tailed). e The length of columnar and mixed BAR-T epithelium decreases upon HOXA13 KO in the rat trachea in vivo tissue reconstitution model. Mean $\pm \mathrm{SEM},{ }^{*} p<0.05$, exact $p=0.0439$.

d Representative examples of H\&E, PAS staining, anti-IVL IHC of BAR-T epithelium from the rat trachea in vivo tissue reconstitution model. H\&E staining shows more layers of cells in animals transplanted with HOXA13+ wild type cells. Periodic acid-Schiff (PAS) stains polysaccaride molecules, and positivity is indicative of goblet-like cells. The arrows point to PAS positivity, which is present in the right panel but not in the left panel, where the BAR-T HOXA13cells are shown. IVL staining is strong in morphologically squamous cells in the left hand panel and weaker in the HOXA13+ epithelium ( $n=3$ for $H O X A^{-}$ and $n=5$ for $\mathrm{HOXA13}^{+}$). $\mathbf{f H O X A 1 3 ^ { - }}$ and HOXA13+ representative pictures of H\&E staining, PAS staining and IVL IHC of the BAR-T organotypic cell culture system indicate that HOXA13 KO reprograms the columnar epithelial phenotype towards squamous keratinized epithelium ( $n=1$ independent experiments).

subjects from the World Medical Association Declaration of Helsinki”. Biopsy specimens to investigate $H O X$ collinearity were obtained by double balloon enteroscopy. Nine biopsy specimens were obtained from each patient $(n=3)$ at different locations along the GI-tract. Sequentially these locations were: esophagus, stomach, duodenum, jejunum, proximal ileum, distal ileum, ascending colon, descending colon and sigmoid/rectum (Supplementary Fig. 2). Included patients had unexplained symptoms, mostly anemia, while inflammatory bowel disease patients were excluded. All biopsies for RNA isolation were stored in RNA-later at $-80^{\circ} \mathrm{C}$. Squamous esophageal biopsies $(n=13)$ originated $5 \mathrm{~cm}$ above the squamocolumnar junction (SCJ). Barrett's (BE) biopsies $(n=13)$ originated caudal of the SCJ and cranial of the gastric folds (all patients were on PPI therapy), stomach biopsies $(n=12)$ were from the corpus. All three types of biopsy specimens were derived from the same patients, (one stomach biopsy specimen was not obtained due to patient agitation during the gastroscopy). The squamous esophageal, $\mathrm{BE}$, and stomach biopsies were taken in a paired fashion from 13 patients. Where the number of samples is indicated below, this indicates the number of individual patients. Forceps biopsy specimens of EACs $(n=12)$ were obtained. Pathological examination of simultaneously taken forceps biopsies around the study specimens had to be positive for EAC. Gastric inlet patch were sampled from proximal esophagus. To determine the proximal colonic HOXA13 border, biopsies were taken from the cecum at the appendix base, the ileocecal valve, $5 \mathrm{~cm}$ distal to the ileocecal valve, and from the transverse colon in each patient $(n=5)$.

Collection of archival pathology specimens. FFPE material was collected from gastric IM (from the antrum, angulus, and corpus, i.e., not from the cardia; $n=12$ ), the gastric inlet patch $(n=5)$, CLE (from the proximal esophagus; $n=14$ ), and Meckel's diverticula $(n=14)$. For RNAscope RNA-ISH, one FFPE specimen of each of these origins was used. Depending on the extent of metaplasia, remainder of tissues was used whole, macroscopically separated, or processed with the PhotoActivated Localization Microscopy with the Laser Caption Microdissection (PALM LCM) for mRNA isolation and subsequent qPCR. Nuclease-free membrane slides treated with UV light at $254 \mathrm{~nm}$ for 30 min were used to mount $10 \mu \mathrm{m}$ sections, dried overnight at $56^{\circ} \mathrm{C}$, deparaffinized, stained with hematoxylin and eosin, and dehydrated. AdhesiveCap microtubes obtained from Zeiss (Oberkochen, Germany) were used to collect the tissue of interest after cutting and pulsing of the PALM LCM. Additionally, FFPE materials or fresh pinch biopsies were collected from the squamous esophagus of a patient without $\mathrm{BE}$, the squamous esophagus of a $\mathrm{BE}$ patient, BE, EAC, stomach (the corpus), and the ileum. Colon was used as a positive control. FFPE materials that were collected only for RNA-ISH were pyloric metaplasia (from the colon; $n=5$ ), Paneth cell metaplasia (from the colon; $n=5$ ), fetal GEJ tissue ( $n=2$ of 17 weeks, and $n=1$ of 20 weeks; this material originated from spontaneous abortions), and adult GEJ tissue consisting out of continuous strips of tissue containing squamous esophageal epithelium, GEJ, and oxyntic stomach epithelium $(n=3)$. Two strips came from surgical specimens without evidence of $\mathrm{BE}$, with a neuroendocrine tumor and decompensated achalasia (male of 71 and female of 56 years old). The third patient had surgery to remove an EAC (male of 63 years old). All tissues were obtained from the gastroenterology and pathology departments of the Erasmus MC according to the FEDERA code of conduct $^{58}$. The use of archival pathology specimens was authorised by an institutional review board (METC -Erasmus MC) on the basis that the material were obtained as left over specimens from routine diagnostics and thus not subject to the WMO (Law Medical Research from the Netherlands) and informed consent was waived by the institutional review board.

Animal studies. For the Hoxa13 mRNA expression analysis throughout the murine gastrointestinal (GI) tract, four $C 57 B L / 6 J$ wildtype mice were used between three and five months of age. The GI-tract was divided into 1: esophagus; 2: stomach; 3: duodenum; 4: jejunum; 5: proximal ileum; 6: distal ileum; 7: cecum; 8: proximal colon; 9: distal colon, of which sections were opened and rinsed in PBS followed by storage in RNAlater at $-80^{\circ} \mathrm{C}$ (Supplementary Fig. 1b). For determining which cells express Hoxa13 in the GI-tract, tissues from a C57BL/6JHoxa13-GFP heterozygous mutant mouse model were employed, in which GFP expression is driven by the endogenous mouse Hoxal3 promotor through the creation of a fusion protein ${ }^{59}$. Mice were generally kept with $12: 12 \mathrm{~h}$ light-dark, the animal room temperature is between 20 and $24 \mathrm{oC}$ and the relative humidity is $55 \pm 10 \%$. These tissues were taken out and embedded in O.C.T. Compound bought from Qiagen Inc. (Hilden, Germany) and frozen at $-80^{\circ} \mathrm{C}$. Cryosections were made which were mounted in fluoroshield mounting medium with DAPI obtained from Abcam (Cambridge, UK). Subsequently, the GEJ and the distal GItract were analyzed directly for GFP expression using the Zeiss confocal laser scan microscope LSM 510. Additionally, immunohistochemistry staining was performed with anti-GFP antibody (\#AB3080, Bio-Connect BV) (see below). These murine experiments were approved by the Ethical Committee for Animal Experiments of the Erasmus MC and were performed according to the guidelines of the same institution.

Immunohistochemistry. For immunohistochemistry, slides were blocked in $10 \%$ of normal goat serum, antigens were retrieved by boiling samples in citrate buffer (pH6), and samples were incubated overnight at $4{ }^{\circ} \mathrm{C}$ with primary antibody. Dilutions and manufactures of primary antibodies are presented in the Supplementary Table 4. After incubation with HRP-conjugated secondary antibody (Dako EnVision+System-HRR labeled Polymer Anti Mouse, Dako) endogenous peroxidase was blocked in $3 \% \mathrm{H}_{2} \mathrm{O}_{2}$ and antibody binding was visualized by DAB staining. IHC analysis for HOXA13 was tried using antibodies ab106503 and ab26084, however these failed to show specificity and have since been discontinued by the companies offering them. H\&E staining was performed by ${ }^{60}$. For H\&E stainings de-parafinized $4 \mu \mathrm{M}$ slides were incubated during $3 \mathrm{~min}$ in hematoxylin solution, followed by tap water washes and $15 \mathrm{~s}$ of incubation with eosin. For PAS staining, de-parafinized slides were incubated with $0.5 \%$ Periodic Acid solution for $10 \mathrm{~min}$, followed by two ddH2O washes and incubation in Schiffs reagent (Sigma Aldrich) for $15 \mathrm{~min}$ and hematoxylin for 3 mins.

Multiplex immunofluorescent staining. Triplex staining for keratin 5, keratin7 and p63 was done by automated multiplex IF using the Ventana Benchmark Discovery (Ventana Medical Systems Inc.). In brief, following deparaffinization and heat-induced antigen retrieval with CC1 (\#950-500, Ventana) for $64 \mathrm{~min}$ at $97^{\circ} \mathrm{C}$, the tissue samples were incubated firstly with Keratin 5 antibodies for 32 min at $37^{\circ} \mathrm{C}$ followed by detection with Ultramap anti-rabbit HRP (\#760-4315, Ventana) for $12 \mathrm{~min}$ followed by visualization with Red610 for 8 min (\#760-245, Ventana). Antibody denaturing was performed using CC2 (\#950-123, Ventana) for $20 \mathrm{~min}$ at $100^{\circ} \mathrm{C}$. Secondly, Keratin 7 antibodies were incubated for $32 \mathrm{~min}$ at $37^{\circ} \mathrm{C}$ followed by detection with Ultramap anti-rabbit HRP (\#760-4315, Ventana) followed by visualization with FAM (\#760-243, Ventana) for $4 \mathrm{~min}$. Antibody denaturing was performed using CC2 (\#950-123, Ventana) for $8 \mathrm{~min}$ at $100^{\circ} \mathrm{C}$. Thirdly, P63 antibodies were incubated for $32 \mathrm{~min}$ at $37^{\circ} \mathrm{C}$ followed by detection with Ultramap anti-mouse HRP (\#760-4313, Ventana) for $12 \mathrm{~min}$ followed by visualization with Cy5 for 12 min (\#760-238, Ventana). Slides were incubated in PBS with DAPI for $15 \mathrm{~min}$ and covered with anti-fading medium (DAKO, S3023).

RNA isolation. RNA was isolated using the NucleoSpin RNA isolation kit (Macherey Nagel, Düren, Germany). Biopsies and animal tissues were homogenized by the TissueRuptor obtained from Qiagen Inc. RNA concentrations were measured using a Nanodrop spectrophotometer and samples were stored in RNA storage solution (Sodium Citrate $\mathrm{pH}$ 6.4), bought from Ambion (Foster City, USA) and kept at $-80^{\circ} \mathrm{C}$. RNA integrity was checked with $1 \%$ agarose gelelectrophoresis. FFPE material was deparaffinized with xylene and ethanol, lysed, digested with proteinase K, and RNA was isolated with the High Pure FFPET RNA isolation kit obtained from Roche (Basel, Switzerland). RNA isolation from dedifferentiated $\mathrm{KH} 2$ mouse embryonic stem cells (mESCs) was done using a picopure RNA isolation kit (Thermo-Fisher Scientific, Waltham, USA). After RNA isolation all samples for RNA-Sequencing were tested on the Agilent 2100 Bioanalyzer to determine RNA integrity and quantity. 
CDNA and qPCR. cDNA was made from $1 \mu \mathrm{g}$ RNA using Primescript RT Master Mix according to manufacturer's instructions (Takara, Otsu, Japan), for $15 \mathrm{~min}$ at $37^{\circ} \mathrm{C}$ and $5 \mathrm{~s}$ at $95^{\circ} \mathrm{C}$, and stored at $-20^{\circ} \mathrm{C}$. qPCR was performed for 40 cycles in the iQ5 Real-Time PCR detection system that was obtained from BioRad Laboratories (Veenendaal, The Netherlands). For each reaction $10 \mu \mathrm{l}$ cDNA template, $12.5 \mu$ SYBR GreenER purchased from Invitrogen (Carlsbad, CA), and $2.5 \mu \mathrm{l}$ $10 \mathrm{pM} / \mu \mathrm{l}$ primer were used. Reactions were performed in duplicate. Primers used are shown in Supplementary Table 2 and were ordered at Sigma-Aldrich (Darmstadt, Germany). qPCR data were analyzed with Microsoft Excel using the $\Delta \Delta \mathrm{Ct}$ method. Reference genes used for PCRs on human materials were RP2, $\beta$-ACTIN, and GAPDH. Reference genes used for PCRs on mice materials were Eef2, Rpl37, and Leng8. Differences in expression were analyzed with a two sided Student's ttest using Prism 5.01, obtained from GraphPad Software (San Diego, USA). Values from individual samples were excluded if they deviated more than 2 SD from the mean. Correlations between HOTTIP expression in the squamous esophagus and $\mathrm{BE}$, and correlations between HOTTIP and HOXA13 expression levels in the squamous esophagus and BE were tested using nonparametric Spearman correlations. This is depicted in graphs by connecting lines between datapoints, also indicating the paired nature of the specimens, i.e. they are derived from the same patient, used for this analysis.

In situ hybridization by RNAscope. RNAscope was performed according to the instructions of the manufacturer of the probes and the reagent kit (VS Reagent Kit 320600; Advanced Cell Diagnostics), on proteinase $\mathrm{K}\left(0.1 \%, 10 \mathrm{~min}\right.$ at $\left.37^{\circ} \mathrm{C}\right)$ treated paraffin sections $(5 \mu \mathrm{m})$. Subsequently, slides were hybridized with the RNA probe from RNAscopeVS Hs-HOXA13, (art. \#ACDA 400226), or the control probe also from RNAscopeVS Hs-PPIB (art. \#ACDA 313901)61. PPIB (peptidylprolyl isomerase B) is a ubiquitously expressed gene. The RNAscope probe Hybridization in situ Multiplex was bought from Advanced Cell Diagnostics (Newark, USA) Pyloric metaplasia and Paneth cell metaplasia of the colon were quantified using FIJI, for which a macro was made (Supplementary method 1$)^{62}$. For illustrations of RNA-Scope slides in the paper, background grey signal reduction was performed using Photoshop.

Analysis of GSE datasets. Expression profiles from clonogenic human gastrointestinal stem cell cultures were obtained from Gene Expression Omnibus datasets GSE57584 $4^{15}$ and GSE65013 ${ }^{18}$. In silico analyses were performed using the NCBI Gene Expression Omnibus (GEO) database. Analyses in the GEO database were performed by using the GEO2R tool (www.ncbi.nlm.nih.gov/geo/geo2r/), R 3.2.3., Biobase 2.30.0, GEOquery 2.40.0, limma $3.26 .8^{63}$. The results were represented as a ${ }^{2} \log$-fold change ( ${ }^{2} \log$-FC). In Microsoft Excel, this ${ }^{2} \log$-FC was converted to fold change (FC). For each ${ }^{2} \log$-FC an empirical Bayes moderated t-statistic was calculated. $p$-values were corrected for multiple testing using the Benjamini \& Hochberg false discovery rate method.

Analysis of single cell RNA seq datasets. BE and ESMG Single Cell Experiment Matrix from supplementary Data files $6 \mathrm{ll}$ three Experiment Matrixes have been mapped to hg38 standard human genome ('TxDb.Hsapiens.UCSC.hg38.knownGene' R-package), normalized as Reads Per Kilobase per Million mapped reads (RPKM). Genes expressed in less than in $0.5 \%$ cells were filtered out. Low-quality cells were excluded based on: (1) the number of expressed genes-for 10x SingleCell sequence data, cells expressing less than 400 or more than 7000 genes, for smartSeq data cells expressing less than 1000 and more than 7000 were removed. Different numbers were chosen due to the different sequencing depth. (2) Boxplot representation of all cells-outliers, i.e. cells mapping higher or lower than $1.5 \mathrm{x}$ the first or third quartile were removed. (3) Based on \% of reads - cells were removed if there were more than $20 \%$ of reads mapping to mitochondrial or ribosomal genes. HOXA13-related genes query: HOXA13-positive cells from normal esophagus were selected with R. Genes that were expressed in at least $70 \%$ of these HOXA13positive cells (20445) were analyzed for their expression in HOXA13-negative cells of normal esophagus as well as HOXA13 negative and positive cells in BE tissue. For T-SNE plot, 638 cells were included (388 cells from Barrett's tissue, 250 cells from normal esophagus) and plotted based on their location of origin (colour) as well as HOXA13 expression (open vs closed symbols).

Cell culture. All cells were cultured with penicillin $(100 \mu / \mathrm{ml})$ and streptomycin $(100 \mu / \mathrm{ml})$ and were regularly STR-verified and checked for mycoplasma by handing in samples prepared according to instructions at GATC Biotech (Konstanz, Germany). Primary human esophageal epithelial cells transformed with hTERT (EPC2-hTERT) (gift of K.K. Krishnadath) ${ }^{64}$, were cultured with Keratinocyte SFM medium, supplemented with bovine pituitary extract at $50 \mu \mathrm{g} / \mathrm{ml}$ and EGF at $1 \mathrm{ng} / \mathrm{ml}$ (Thermo-Fisher Scientific). HET1A, the primary immortalized human squamous esophageal cell line Het-1A was a gift of J.W.P.M. van Baal (University Utrecht, The Netherlands). These cells were grown in EPM2 medium obtained from AthenaES, (Baltimore, Maryland, USA). The primary immortalized human BE cell line (BAR-T) was a gift of dr. J.W.P.M. van Baal who had, in turn, received them from dr. R.F. Souza (University of Texas Southwestern Medical Center, USA). These cells were grown in supplemented keratinocyte basal medium (KBM2), bought from Lonza (Basel, Switzerland), according to the method of
Jaiswal et al. ${ }^{65}$. KH2 mESCs were a gift of J. Gribnau and maintained in DMEM with $10 \%$ FCS, Non-Essential Amino Acids, sodium pyruvate, LIF, and $\beta$ mercapto-ethanol (embryonic stem cell medium; Supplementary Table 3). Dishes were coated with attachment factor protein solution (Thermo-Fisher Scientific). Irradiated mouse embryonic fibroblasts (3T3-Swiss albino cells (gift of J.W.P.M. van Baal), cultured in DMEM with 10\% FCS, were used as feeder cells. HEK293T cells were cultured in DMEM with 10\% FCS.

Generation of EPC2-hTERT HOXA13 overexpression model. The human HOXA13 gene including its single intron was amplified using Q5 polymerase from gDNA using primers (AgeI HoxA13 F; GGTGGTACCGGTGCCACCATGACA GCCTCCGTGCTCCT, and XbaI HoxA13 R; ACCACCTCTAGATTAACT AGTGGTTTTCAGTT) and cloned into pEN_TmiRc3 using AgeI and XbaI restriction sites, a gift from Iain Fraser (Addgene (Cambridge, USA) $\# 25748)^{66}$ Subsequently, the HOXA13 insert was transferred into pSLIK-Venus, using a Gateway reaction ${ }^{67}$. pSLIK-Venus was a gift from Iain Fraser (Addgene \#25734) ${ }^{66}$ A similar plasmid but without the HOXA13 insert served as control. Both plasmids were sequenced by LGC Genomics (Teddington, UK). Next, plasmids were packaged into lentiviral particles following transfection in HEK293T cells with third generation packaging plasmids. The supernatant was collected and ultracentrifuged. EPC2-hTERT cells were transduced with the virus and FluorescenceActivated Cell Sorted (FACS) for YFP (pSLIK-Venus) positive cells on the BD FACSCantoTM II that was bought from BD Biosciences (San Jose, USA). These cells were grown and analyzed as a cell pool. HOXA13 was induced by the addition of $1,25 \mu \mathrm{g} / \mathrm{ml}$ doxycycline to the culture medium. Overexpression was determined by $\mathrm{qPCR}$ according to scientific standards ${ }^{50}$.

Generation of KH2 embryonic stem cells HOXA13 overexpression model. The human HOXA13 gene including its single intron was amplified using Q5 polymerase from gDNA using primers with an added N-terminal FLAG-tag sequence (GACTACAAAGACGATGACGACAAG) and Kozak sequence (GCCGCCACC; Supplementary Table 3). Next, this PCR product was ligated into EcoRI digested pgk-ATG-frt (Addgene \#20734) using Gibson Cloning (New England BioLabs Inc., Ipswich, USA). pgk-ATG-frt was a gift from Rudolf Jaenisch ${ }^{68}$. KH2 mESCs were passaged the day before the electroporation and four hours before electroporation medium was replaced. Approximately $1.510^{7} \mathrm{KH} 2$ cells were electroporated with $50 \mu \mathrm{g}$ of pgk-ATG-frt-HOXA13 and $25 \mu \mathrm{g}$ of pCAGGS-FLPe-puro (Addgene $\# 20733)^{69}$. Cells were electroporated in $4 \mathrm{~mm}$ cuvettes, with two consecutive pulses ( $400 \mathrm{~V} / 250 \mu \mathrm{F}$ ) using a Gene PulserXcell (Bio-Rad Laboratories). The next day 140 $\mu \mathrm{g} / \mathrm{ml}$ Hygromycin B (Thermo-Fisher Scientific) was added for the selection of correctly targeted colonies. DNA from resistant colonies was isolated with the Kleargene XL blood DNA extraction kit (LGC, Teddington, UK) and analyzed by Q5 PCR using the following primers: PGK-F1 or PGK-F2 and T1E2-HygroR6 and T1E2-HygroR7 (Supplementary Table 3). Correctly-targeted clones were checked for proper HOXA13 induction by the addition of $1.25 \mu \mathrm{g} / \mathrm{ml}$ doxycycline to the culture medium for 3 days. Three HOXA13 overexpression versus three control biological replicates were selected and used for experiments.

Differentiation of KH2 mouse embryonic stem cells. An optimized version of the Ogaki protocol was used ${ }^{70}$. Cells were plated on $50 \%$ confluent pre-cultured M15 cells, a mesoderm-derived feeder cell line ${ }^{71}$ (gift of N. Hastie, University of Edinburgh, UK). Cells grew six days in differentiation medium consisting of ESC medium without LIF, with the addition of Activin-A, basic Fibroblast Growth Factor, CHIR, and Noggin (Supplementary Table 3). HOXA13-expression was induced on day four using doxycycline at $1.25 \mu \mathrm{g} / \mathrm{ml}$. On day six, cells were analyzed by FACS by double staining with $0.8 \mu \mathrm{g}$ PE Rat Anti-Mouse CD184 (CXCR4) and $2.0 \mu \mathrm{g}$ Anti-CD324 Alexa Fluor ${ }^{\oplus} 488$ (E-Cadherin) at $4{ }^{\circ} \mathrm{C}$ for $45 \mathrm{~min}$ (Supplementary Table 3). The cells were analysed with a BD FACSCantoTM II (BD Biosciences, USA). Data were analyzed with BD FACSDiva v8.0.1 software, which was obtained from BD Biosciences, and processed using Microsoft Excel. Example of analysis is provided in the Supplementary Fig. 11. Double-positive cells were sorted and cultured for another day with doxycycline at $1.25 \mu \mathrm{g} / \mathrm{ml}$ before harvesting and RNA isolation took place, using the picopure RNA isolation kit (Thermo-Fisher Scientific).

Generation of the BAR-T HOXA13 knock-out model. Functional HOXA13 was removed from BAR-T cells using CRISPR/Cas9-mediated gene editing. A HOXA13 sgRNA targeting exon 1 was cloned into pTLCV2, by ligating two annealed oligonucleotides, i.e. Guide1sgRNA F and R (Supplementary Table 4). TLCV2 was a gift from Adam Karpf (Addgene \#87360) ${ }^{72}$. Following sequence verification, the pTLCV2-HOXA13sgRNA plasmid was packaged into lentiviral particles by cotransfection into HEK293T cells with pSPAX2 and pMD2.G, gifts from Didier Trono (Addgene \#12260 and \#12259). The supernatant was harvested and ultracentrifuged after which BAR-T cells were transduced. Mixed populations of transduced cells were plated at very low confluence, single cell clones could subsequently be isolated using glass cloning cylinders and low melting point agarose from Sigma-Aldrich, followed by DNA isolation using the Kleargene kit, followed by sequence verification with primers TILHOXA13R3 and Pre-HOXA13-FW2 flanking the sgRNA-site (Supplementary Table 4). Three cell lines in which both 
alleles were affected by unique out-of-frame deletions were selected along with three control cell lines.

RNA-sequencing. The EPC2-hTERT samples $(n=8)$ were treated with the TruSeq Stranded mRNA Library Prep Kit. Sequencing took place according to the Illumina TruSeq v3 protocol on an Illumina HiSeq2500 sequencer. Sample preparation and sequencing was performed at the Erasmus MC. Reads of 50 base-pairs were generated and mapped against reference genome hg19 with Tophat (version 2.0.10). Expression was quantified using HTseq-count (0.6.1). Stranded libraries of the BAR-T $(n=6)$, and both non-differentiated and differentiated KH2 mESCs ( $n=6$ each) were prepared with the NEBNext RNA Ultra sample prep kit. Sequencing took place according to the Illumina NestSeq 500 protocol on an Illumina HiSeq2500 sequencer. Sample preparation and sequencing was performed at GenomeScan in Leiden, The Netherlands. Reads of 75 base-pairs were generated, mapped against reference genome hg19 or $\mathrm{mm} 9$ with Tophat (version 2.1.0), and quantified using HTSeq (version 0.6.1p1). Data were processed using R. version $3.2 .5^{73}$, in combination with the module DeSeq2 ${ }^{74}$. Generated FCs and p-values adjusted for multiple testing, i.e. $q$-values, were analyzed using Ingenuity Pathway Analysis (IPA) version 42012434, obtained from Qiagen Inc. (Hilden, Germany) ${ }^{75}$. We limited the number of genes analyzed to a maximum of 1000 by eliminating genes with a (relatively) low fold change if differentially expressed genes number was above 1000 . The dataset cut-offs used were always a $q$ value of 0.05 , the fold change cut-off was set at: nondifferentiated KH2-mESCs, FC 2, 888 genes; differentiation of KH2-mESCs, FC 5, 924 genes; differentiated KH2-mESCs, FC not restricted, 665 genes; BAR-T, FC not restricted, 146 genes; EPC2-hTERT, FC 1.3, 990 genes. Activity scores are known in IPA as " $z$-scores" which represents the number of standard deviations from the mean of a normal distribution. For analysis and visualization of gene expression in the epidermal differentiation complex the raw counts from both models normalized to total reads were used. Genes for which one of both cell models had less than ten reads in the control or experimental samples were excluded. Overlap in multiple testing corrected differentially expressed genes in the BAR-T and EPC2-hTERT datasets was calculated as follows; the proportion of overexpressed genes in the EPC2-hTERT dataset was determined. Half of the differentially expressed genes in the BAR-T dataset would be expected to be regulated in the same direction if regulation would be random. This expected overlap if regulation was random, and the observed overlap, were used as input for an $\mathrm{X}^{2}$ test. Information included in Supplementary Data 2 and 3 in the "known function" and "Detailed description" columns was obtained through nonsystematic review and should not be considered as an exhaustive overview of the literature. Association of expression of molecules in the distal GI-tract with their regulation by HOXA13 expression was reviewed using the human proteome atlas and depicted in Supplementary Data $2^{32}$.

Acid and bile exposure. For assessment of HOXA13 mRNA expression upon acid/ bile exposure, EPC2-hTERT and HET-1A cells were treated for $30 \mathrm{~min}$ with cell culture medium adjusted to a $\mathrm{pH}$ of 7.0 or 4.0 using $\mathrm{HCl}$. Cells were subsequently washed using PBS and given standard medium. Acid experiments were performed four times in duplo. Cells were separately exposed to medium with a bile acid mixture in concentrations of 0,200 , (and 400 for EPC2-hTERT) $\mu$ mole/L for 30 $\mathrm{min}$ at a $\mathrm{pH}$ of 7.0 . The bile acid mixture consisted of $25 \%$ deoxycholic acid, $45 \%$ glycocholic acid and 30\% taurochenodeoxycholic acid. Cells were subsequently washed using PBS and given normal medium. Bile experiments were performed twice in duplicate. After $24 \mathrm{~h}$, the cells were harvested and RNA was isolated. Methods were derived from Bus et al. ${ }^{76}$. To assess the effect of bile/acid on expansion of cells, EPC2-hTERT cells transduced with HOXA13 or control vector as described above were seeded in 96-well plate with at least 2 wells per condition. Next day, medium was replaced with $100 \mu$ lof bile/acid mixture in cell culture medium $(50 \mu \mathrm{M}$ of sodium glycocholatenhydrate, $50 \mu \mathrm{M}$ taurochenodeoxycholic acid, $\mathrm{pH}=4.95)$. After incubation for 4 days, MTT test was performed as described below. Experiment was performed at least five times.

BAR-T spatial distribution experiments. These were performed with three biological replicate cell lines containing HOXA13 knock-out and three control cell lines. 40.000 BAR-T cells were seeded in a 6 well plate and pictures were taken the second day after seeding. Per well three pictures were taken. These pictures were analyzed using FIJI, using the multipoint tool, an X and Y (pixel) coordinate table was generated ${ }^{62}$. The distance between each cell and its three closest neighbors was quantified using Microsoft Excel and analyzed by two sided student's t-test. The experiment was performed in three independent cell lines and repeated three times.

MTT assay. For assessment of cell growth of EPC2 and BAR-T cells, we performed a 3-(4,5-dimethylthiazol-2-yl)-2,5-diphenyltetrazolium bromide (MTT) assay77. We seeded 1000 cells per well in 96 well plates for each of the three wild-type and three HOXA13 knock-out cell lines. Per condition at least 2 wells were used. On days one, three, five, and seven $10 \mu \mathrm{l} \mathrm{MTT}$ at $5 \mu \mathrm{g} / \mathrm{ml}$ was added and incubated for three hours, the medium was removed, and the precipitate was dissolved in $100 \mu \mathrm{l}$ DMSO, which was incubated for five minutes under continuous shaking. For BAR$\mathrm{T}$ cells, absorption was measured in a BioRad microplate reader Model $680 \mathrm{XR}$ at 490 and $595 \mathrm{~nm}$, the average absorption was used to process the data. For EPC2 cells it was measured with Tecan microplate reader Model Infinite 200 pro at 565 $\mathrm{nm}$ with reference wavelength $670 \mathrm{~nm}$. The experiment was repeated three times and a two sided Student's t-test was used to test for statistical significance.

3D culture EPC2-hTERT cells. 3D culturing of EPC2-hTERT cells was performed as previously described ${ }^{48}$. 4000 EPC2-hTERT cells in culture medium were mixed 1: with ice-cold Matrigel basement membrane matrix (Corning BV), seeded in $50 \mu \mathrm{l}$ drops in a 24 well plate for cell suspension, and incubated at $37^{\circ} \mathrm{C}$ for $30 \mathrm{~min}$. After solidification, $500 \mu \mathrm{l}$ of culturing medium supplemented with $0.6 \mathrm{mM} \mathrm{CaCl} 2$ was added. Y27632 $(10 \mu \mathrm{M})$ was included in medium only the first $24 \mathrm{~h}$ after seeding Medium was refreshed and pictures were taken every three days. The morphology of spheroids (based on number of extrusions, or 'invadosomes') was counted on day 5 . The area of the spheroids was measured with FIJ ${ }^{60}$. For H\&E staining and IHC analysis of involucrin (see above), spheroids were fixed in $4 \%$ formaldehyde for $7 \mathrm{~min}$ on day 11 , washed with PBS, put in $2 \%$ agarose, and embedded in paraffin, then $4 \mu \mathrm{M}$ slices were sectioned. Quantification was based on the percentage of positive cells and the intensity of the staining (scores ranged from 0, 2 to 9).

Organotypic air-liquid interface culture. Plate inserts (Sigma-Aldrich, Germany) were covered with bovine collagen I (Thermo Fisher Scientific, USA). The fibroblast (3T3-Swiss albino) feeder layer was embedded within a collagen matrix and was allowed to mature for 7 days, after which time BAR-T HOXA13 knock-out and control epithelial cells were seeded on top and allowed to grow to confluence for an additional 3 days as described ${ }^{78}$. Then the culture media level of the upper well was reduced, exposing the apical side of keratinocytes to the air, while maintaining liquid levels at the basolateral side. On day 15 , cultures were harvested for histologic examination. $4 \mu \mathrm{M}$ paraffin-embedded sections were deparaffinized, and staining with hematoxylin and eosin, PAS staining and immunohistochemistry for involucrin were performed.

Rat trachea in vivo tissue reconstitution model. 500,000 parental BAR-T cells (derived from six independent clones) or HOXA13 knock-out clones (three independent clones) in $30 \mu \mathrm{l}$ of medium were sealed in the lumen of devitalized and denuded rat tracheas and implanted under the dorsal skin of NOD SCID gamma mice as described by Croagh et al. ${ }^{79}$. Mice were housed in microisolator cages with a $14 \mathrm{~h}$ light $/ 10 \mathrm{~h}$ dark cycle, standard chow and water ad libitum, and temperature and humidity maintained at $21 \pm 1{ }^{\circ} \mathrm{C}$ and $50 \pm 10 \%$, respectively. Mice were sacrificed after four or six weeks. Harvested rat tracheas were formalin fixed, decalcified, embedded in paraffin and sectioned. Staining with hematoxylin and eosin, alcian blue, PAS staining and immunohistochemistry using antibodies against human mitochondria, CK7, TFF3, CDX2, p63, CK5, and involucrin (a gift from Prof. Pritinder Kaur, Curtin University, Australia or \#19018-100UL from Sigma-Aldrich) were performed (Supplementary Fig. 9 and Table 4). These murine experiments were approved by the Peter MacCallum Cancer Centre Animal Experimentation Ethics Committee and were performed according to the guidelines of the same institution.

Reporting summary. Further information on research design is available in the Nature Research Reporting Summary linked to this article.

\section{Data availability}

The RNA sequence data discussed in this publication have been deposited in NCBI's Gene Expression Omnibus ${ }^{80}$ and are accessible through GEO Series accession number GSE173170 ${ }^{81}$. There are no restrictions regarding data availability. Supplemental figures, tables, and a method are included. Source data are provided with this paper and all relevant data are available from the authors.

Datasets used in the manuscript: RNA seq data GSE57584 ${ }^{15}$, GSE65013 $3^{18}$, single cell RNA data $\mathrm{seq}^{21}$, GSE134520 $0^{82}$, GSE $81861^{83}$. Source data are provided with this paper.

Received: 21 December 2018; Accepted: 6 May 2021; Published online: 07 June 2021

\section{References}

1. Grotenhuis, B. A. et al. Delay in diagnostic workup and treatment of esophageal cancer. J. Gastrointest. Surg. 14, 476-483 (2010).

2. Thrift, A. P. \& Whiteman, D. C. The incidence of esophageal adenocarcinoma continues to rise: analysis of period and birth cohort effects on recent trends. Ann. Oncol. 23, 3155-3162 (2012).

3. Ferlay, J. et al. Cancer incidence and mortality worldwide: sources, methods and major patterns in GLOBOCAN 2012. Int. J. Cancer 136, E359-E386 (2015)

4. Thirunavukarasu, P. et al. Meckel's diverticulum-a high-risk region for malignancy in the ileum. Insights from a population-based epidemiological study and implications in surgical management. Ann. Surg. 253, 223-230 (2011). 
5. Orosey, M., Amin, M. \& Cappell, M. S. A 14-year study of 398 esophageal adenocarcinomas diagnosed among 156,256 EGDs performed at two large hospitals: an inlet patch is proposed as a significant risk factor for proximal esophageal adenocarcinoma. Dig. Dis. Sci. 63, 452-465 (2017).

6. Barros, R. et al. Dynamics of SOX2 and CDX2 expression in Barrett's mucosa. Dis. Markers 2016, 1532791 (2016).

7. Mari, L. et al. A pSMAD/CDX2 complex is essential for the intestinalization of epithelial metaplasia. Cell Rep. 7, 1197-1210 (2014).

8. Silberg, D. G. et al. Cdx2 ectopic expression induces gastric intestinal metaplasia in transgenic mice. Gastroenterology 122, 689-696 (2002).

9. di Pietro, M. et al. Evidence for a functional role of epigenetically regulated midcluster HOXB genes in the development of Barrett esophagus. Proc. Natl Acad. Sci. USA 109, 9077-9082 (2012).

10. Pearson, J. C., Lemons, D. \& McGinnis, W. Modulating Hox gene functions during animal body patterning. Nat. Rev. Genet. 6, 893-904 (2005).

11. Shah, N. \& Sukumar, S. The Hox genes and their roles in oncogenesis. Nat. Rev. Cancer 10, 361-371 (2010).

12. Kawazoe, Y. et al. Region-specific gastrointestinal Hox code during murine embryonal gut development. Dev. Growth Differ. 44, 77-84 (2002).

13. Yahagi, N. et al. Position-specific expression of Hox genes along the gastrointestinal tract. Congenit. Anom. 44, 18-26 (2004).

14. Wang, K. C. et al. A long noncoding RNA maintains active chromatin to coordinate homeotic gene expression. Nature 472, 120-124 (2011).

15. Wang, X. et al. Cloning and variation of ground state intestinal stem cells. Nature 522, 173-178 (2015).

16. Gupta, R. A. et al. Long noncoding RNA HONTAIR reprograms chromatin state to promote cancer metastasis. Nature 464, 1071-1076 (2010).

17. Que, J., Garman, K. S., Souza, R. F. \& Spechler, S. J. Pathogenesis and cells of origin of Barrett's esophagus. Gastroenterology 157, 349-364.e341 (2019).

18. Yamamoto, Y. et al. Mutational spectrum of Barrett's stem cells suggests paths to initiation of a precancerous lesion. Nat. Commun. 7, 10380 (2016).

19. Gaunt, S. J. The significance of Hox gene collinearity. Int J. Dev. Biol. 59, 159-170 (2015).

20. Symonds, D. A. Paneth cell metaplasia in diseases of the colon and rectum. Arch. Pathol. 97, 343-347 (1974).

21. Owen, R. P. et al. Single cell RNA-seq reveals profound transcriptional similarity between Barrett's oesophagus and oesophageal submucosal glands. Nat. Commun. 9, 4261 (2018).

22. Jiang, M. et al. Transitional basal cells at the squamous-columnar junction generate Barrett's oesophagus. Nature 550, 529-533 (2017).

23. Krüger, L. et al. Ductular and proliferative response of esophageal submucosal glands in a porcine model of esophageal injury and repair. Am. J. Physiol.Gastrointest. Liver Physiol. 313, G180-G191 (2017).

24. Rotterdam, H. Pathology of the gastric cardia. Verh. Dtsch Ges. Pathol. 83, 37-42 (1999).

25. Johansson, J. et al. Pancreatic acinar metaplasia in the distal oesophagus and the gastric cardia: prevalence, predictors and relation to GORD. J. Gastroenterol. 45, 291-299 (2010).

26. Matsushita, S., Ishii, Y., Scotting, P. J., Kuroiwa, A. \& Yasugi, S. Pre-gut endoderm of chick embryos is regionalized by 1.5 days of development. Dev. Dyn. 223, 33-47 (2002).

27. Boyer, L. A. et al. Core transcriptional regulatory circuitry in human embryonic stem cells. Cell 122, 947-956 (2005).

28. Shin, M. R., Cui, X. S., Jun, J. H., Jeong, Y. J. \& Kim, N. H. Identification of mouse blastocyst genes that are downregulated by double-stranded RNAmediated knockdown of Oct-4 expression. Mol. Reprod. Dev. 70, 390-396 (2005).

29. Voorneveld, P. W. et al. The BMP pathway either enhances or inhibits the Wnt pathway depending on the SMAD4 and p53 status in CRC. Br. J. Cancer 112, 122-130 (2015).

30. Bakre, M. M. et al. Generation of multipotential mesendodermal progenitors from mouse embryonic stem cells via sustained Wnt pathway activation. J. Biol. Chem. 282, 31703-31712 (2007).

31. Denans, N., Iimura, T. \& Pourquie, O. Hox genes control vertebrate body elongation by collinear Wnt repression. Elife 4 https://doi.org/10.7554/ eLife.04379 (2015).

32. Uhlen, M. et al. Proteomics. Tissue-based map of the human proteome. Science 347, 1260419 (2015).

33. Jaiswal, K. R. et al. Characterization of telomerase-immortalized, nonneoplastic, human Barrett's cell line (BAR-T). Dis. Esophagus 20, 256-264 (2007).

34. Mischke, D., Korge, B. P., Marenholz, I., Volz, A. \& Ziegler, A. Genes encoding structural proteins of epidermal cornification and S100 calciumbinding proteins form a gene complex ("epidermal differentiation complex") on human chromosome 1q21. J. Invest. Dermatol. 106, 989-992 (1996).

35. Boczonadi, V. \& Maatta, A. Annexin A9 is a periplakin interacting partner in membrane-targeted cytoskeletal linker protein complexes. FEBS Lett. 586, 3090-3096 (2012).
36. Dai, Y. et al. Genome-wide analysis of Barrett's adenocarcinoma. A first step towards identifying patients at risk and developing therapeutic paths. Transl. Oncol. 11, 116-124 (2017).

37. Kalinin, A., Marekov, L. N. \& Steinert, P. M. Assembly of the epidermal cornified cell envelope. J. Cell Sci. 114, 3069-3070 (2001).

38. Lundstrom, A. \& Egelrud, T. Stratum corneum chymotryptic enzyme: a proteinase which may be generally present in the stratum corneum and with a possible involvement in desquamation. Acta Derm. Venereol. 71, 471-474 (1991).

39. Sun, G., Zhao, G., Lu, Y., Wang, Y. \& Yang, C. Association of EMP1 with gastric carcinoma invasion, survival and prognosis. Int. J. Oncol. 45, 1091-1098 (2014)

40. de Koning, P. J. et al. Downregulation of SERPINB13 expression in head and neck squamous cell carcinomas associates with poor clinical outcome. Int. J. Cancer 125, 1542-1550 (2009).

41. Kimchi, E. T. et al. Progression of Barrett's metaplasia to adenocarcinoma is associated with the suppression of the transcriptional programs of epidermal differentiation. Cancer Res. 65, 3146-3154 (2005).

42. Chaves, P. et al. Chromosomal analysis of Barrett's cells: demonstration of instability and detection of the metaplastic lineage involved. Mod. Pathol. 20, 788-796 (2007).

43. Maru, D. M. et al. Frequent loss of heterozygosity of chromosome 1q in esophageal adenocarcinoma: loss of chromosome 1q21.3 is associated with shorter overall survival. Cancer 115, 1576-1585 (2009).

44. $\mathrm{Li}$, J. et al. Allelic imbalance of chromosome $1 \mathrm{q}$ in esophageal squamous cell carcinomas from China: a novel region of allelic loss and significant association with differentiation. Cancer Lett. 220, 221-230 (2005).

45. Luthra, M. G. et al. Decreased expression of gene cluster at chromosome 1q21 defines molecular subgroups of chemoradiotherapy response in esophageal cancers. Clin. Cancer Res. 13, 912-919 (2007).

46. Quante, M. et al. Bile acid and inflammation activate gastric cardia stem cells in a mouse model of Barrett-like metaplasia. Cancer Cell 21, 36-51 (2012).

47. Menke, V. et al. Conversion of metaplastic Barrett's epithelium into postmitotic goblet cells by gamma-secretase inhibition. Dis. Model Mech. 3, 104-110 (2010).

48. Kasagi, Y. et al. The esophageal organoid system reveals functional interplay between notch and cytokines in reactive epithelial changes. Cell Mol. Gastroenterol. Hepatol. 5, 333-352 (2018).

49. Bajpai, M. et al. Repeated exposure to acid and bile selectively induces colonic phenotype expression in a heterogeneous Barrett's epithelial cell line. Lab Invest 88, 643-651 (2008).

50. Nesteruk, K. et al. Forced expression of HOXA13 confers oncogenic hallmarks to esophageal keratinocytes. Biochimica et. Biophysica Acta (BBA) Mol. Basis Dis. 1866, 165776 (2020).

51. Nesteruk, K., Spaander, M. C. W., Leeuwenburgh, I., Peppelenbosch, M. P. \& Fuhler, G. M. Achalasia and associated esophageal cancer risk: What lessons can we learn from the molecular analysis of Barrett's-associated adenocarcinoma? Biochim. Biophys. Acta Rev. Cancer. 1872, 188291 (2019).

52. Hamilton, S. R. \& Yardley, J. H. Regnerative of cardiac type mucosa and acquisition of Barrett mucosa after esophagogastrostomy. Gastroenterology $\mathbf{7 2}$, 669-675 (1977).

53. Gu, Z. D. et al. HOXA13 promotes cancer cell growth and predicts poor survival of patients with esophageal squamous cell carcinoma. Cancer Res. 69, 4969-4973 (2009).

54. Qin, Z. et al. Elevated HOXA13 expression promotes the proliferation and metastasis of gastric cancer partly via activating Erk1/2. OncoTargets Ther. 12, 1803-1813 (2019)

55. Deng, Y. et al. The expression of HOXA13 in lung adenocarcinoma and its clinical significance: a study based on The Cancer Genome Atlas, Oncomine and reverse transcription-quantitative polymerase chain reaction. Oncol. Lett. 15, 8556-8572 (2018).

56. Quagliata, L. et al. High expression of HOXA13 correlates with poorly differentiated hepatocellular carcinomas and modulates sorafenib response in in vitro models. Lab Invest 98, 95-105 (2018).

57. Dong, Y. et al. HOXA13 is associated with unfavorable survival and acts as a novel oncogene in prostate carcinoma. Future Oncol. 13, 1505-1516 (2017).

58. Federa, Human Tissue and Medical Research: Code of conduct for responsible use. https://www.bbmri.nl/sites/bbmri/files/styles/Federa_code_of_conduct_english.pdf (2011).

59. Perez, W. D., Weller, C. R., Shou, S. \& Stadler, H. S. Survival of Hoxa13 homozygous mutants reveals a novel role in digit patterning and appendicular skeletal development. Dev. Dyn. 239, 446-457 (2010).

60. Schindelin, J. et al. Fiji: an open-source platform for biological-image analysis Nat. Methods 9, 676-682 (2012).

61. Wang, F. et al. RNAscope: a novel in situ RNA analysis platform for formalinfixed, paraffin-embedded tissues. J. Mol. Diagn. 14, 22-29 (2012). 
62. Schindelin, J., Rueden, C. T., Hiner, M. C. \& Eliceiri, K. W. The ImageJ ecosystem: an open platform for biomedical image analysis. Mol. Reprod. Dev. 82, 518-529 (2015).

63. Barrett, T. et al. NCBI GEO: archive for functional genomics data sets-update. Nucleic Acids Res 41, D991-D995 (2013).

64. Harada, H. et al. Telomerase induces immortalization of human esophageal keratinocytes without p16INK4a inactivation. Mol. Cancer Res. 1, 729-738 (2003).

65. Jaiswal, K. R. et al. Characterization of telomerase-immortalized, non neoplastic, human Barrett's cell line (BAR-T). Diseases of the esophagus: official journal of the International Society for. Dis. Esophagus / ISDE 20 , 256-264 (2007).

66. Shin, K. J. et al. A single lentiviral vector platform for microRNA-based conditional RNA interference and coordinated transgene expression. Proc. Natl Acad. Sci. USA 103, 13759-13764 (2006).

67. Katzen, F. Gateway $((\mathrm{R}))$ recombinational cloning: a biological operating system. Expert Opin. Drug Disco. 2, 571-589 (2007).

68. Beard, C., Hochedlinger, K., Plath, K., Wutz, A. \& Jaenisch, R. Efficient method to generate single-copy transgenic mice by site-specific integration in embryonic stem cells. Genesis 44, 23-28 (2006).

69. Buchholz, F., Angrand, P. O. \& Stewart, A. F. Improved properties of FLP recombinase evolved by cycling mutagenesis. Nat. Biotechnol. 16, 657-662 (1998).

70. Ogaki, S., Shiraki, N., Kume, K. \& Kume, S. Wnt and Notch signals guide embryonic stem cell differentiation into the intestinal lineages. Stem Cells $\mathbf{3 1}$, 1086-1096 (2013).

71. Larsson, S. H. et al. Subnuclear localization of WT1 in splicing or transcription factor domains is regulated by alternative splicing. Cell $\mathbf{8 1}$, 391-401 (1995).

72. Sanjana, N. E., Shalem, O. \& Zhang, F. Improved vectors and genome-wide libraries for CRISPR screening. Nat. Methods 11, 783-784 (2014).

73. R Development Core Team R: A language and environment for statistical computing (Vienna, Austria; 2008).

74. Love, M. I., Huber, W. \& Anders, S. Moderated estimation of fold change and dispersion for RNA-seq data with DESeq2. Genome Biol. 15, 550 (2014).

75. Kramer, A., Green, J., Pollard, J. Jr. \& Tugendreich, S. Causal analysis approaches in Ingenuity Pathway Analysis. Bioinformatics 30, 523-530 (2014).

76. Bus, P., Siersema, P. D., Verbeek, R. E. \& van Baal, J. W. P. M. Upregulation of miRNA-143, $-145,-192$, and -194 in esophageal epithelial cells upon acidic bile salt stimulation. Dis. Esophagus 27, 591-600 (2013).

77. Queiroz, K. C. et al. Violacein induces death of resistant leukaemia cells via kinome reprogramming, endoplasmic reticulum stress and Golgi apparatus collapse. PLoS ONE 7, e45362 (2012).

78. Kalabis, J. et al. Isolation and characterization of mouse and human esophageal epithelial cells in 3D organotypic culture. Nat. Protoc. 7, 235 (2012).

79. Croagh, D. et al. Reconstitution of stratified murine and human oesophageal epithelia in an in vivo transplant culture system. Scand. J. Gastroenterol. 43, 1158-1168 (2008).

80. Edgar, R., Domrachev, M. \& Lash, A. E. Gene Expression Omnibus: NCBI gene expression and hybridization array data repository. Nucleic Acids Res. 30, 207-210 (2002).

81. Janmaat V. T. et al. HOXA13 in etiology and oncogenic potential of Barrett's esophagus. NCBI GEO database, accession GSE173170, https://www.ncbi.nlm. nih.gov/geo/query/acc.cgi?acc=GSE173170 (2021).

82. Zhang, P. et al. Dissecting the single-cell transcriptome network underlying gastric premalignant lesions and early gastric cancer. Cell Rep. 27, 1934-1947 e1935 (2019)

83. Li, H. et al. Author Correction: reference component analysis of single-cell transcriptomes elucidates cellular heterogeneity in human colorectal tumors. Nat. Genet. 50, 1754 (2018).

\section{Acknowledgements}

We would like to acknowledge H.F.B.M. Sleddens, H. Stoop, M.H.W. van Dullemen, P. Vasic, I.T.A. Edelijn, M.J. van der Lee, P.J. Zwalua, W.W. van Dam, E. Zielhuis, J. Knoop, M. Doukas, A.L. Nigg and T.P.P. van den Bosch, Erasmus MC - University Medical Center Rotterdam, for their involvement in investigation, W.N.M. Dinjens for providing material, and F. McKeon, The Jackson Laboratory for Genomic Medicine, for conceptualization and providing resources. FAPESP n. 2016/01139-0; 2017/01046-5 for funding.

M.Magierowski was supported by a grant from National Science Centre (Poland): UMO-2016/23/D/NZ4/01913.

\section{Author contributions}

Conceptualization, V.T.J., M.P.P., A.W., J.C., E.J.K., L.J.W.L., W.A.P., M.C.W.S., K.N., G.F. and R.S.; Methodology, V.T.J., G.M.F., M.P.P., R.A.S., N.C., R.S., K.N.; Formal Analysis, V.T.J., K.N., R.A.S.; Investigation, V.T.J., R.A.S., N.C., K.N.; Resources, M.C.W.S., H.S.S., E.M.T., T.S.G.; Writing - Original Draft, V.T.J., K.N. G.M.F. and A.P.V.; Writing - Review \& Editing V.T.J., K.N., M.P.P., M.C.W.S, A.P.V., A.W., G.M.F., R.A.S, R.S., M.J.B., N.C., W.A.P., J.C., E.J.K., L.J.W.L., H.S.S., E.M.T., T.S.G., J.C., M.M., E.J.K., L.J.W.L., K.N.; Funding Acquisition, M.C.W.S, M.P.P, M.J.B., V.T.J., W.A.P.; Visualization, V.T.J., N.C., A.P.V., K.N.; Supervision, M.P.P, M.J.B., M.C.W.S., G.M.F.

\section{Competing interests}

M.J.B.: Cook Medical; consultant, support for industry and investigator initiated studies Boston Scientific; consultant, support for industry and investigator initiated studies. Pentax Medical; support for investigator initiated studies. Mylan; support for investigator initiated studies. ChiRoStim; support for investigator initiated studies. $3 \mathrm{M}$; support for investigator initiated studies. The remaining authors declare no competing interests.

\section{Additional information}

Supplementary information The online version contains supplementary material available at https://doi.org/10.1038/s41467-021-23641-8.

Correspondence and requests for materials should be addressed to M.P.P.

Peer review information Nature Communications thanks Joszef Zàkàny, Rebecca Fitzgerald, Raquel Almeida and the other anonymous reviewer(s) for their contribution to the peer review of this work. Peer reviewer reports are available.

Reprints and permission information is available at http://www.nature.com/reprints

Publisher's note Springer Nature remains neutral with regard to jurisdictional claims in published maps and institutional affiliations.

Open Access This article is licensed under a Creative Commons Attribution 4.0 International License, which permits use, sharing, adaptation, distribution and reproduction in any medium or format, as long as you give appropriate credit to the original author(s) and the source, provide a link to the Creative Commons license, and indicate if changes were made. The images or other third party material in this article are included in the article's Creative Commons license, unless indicated otherwise in a credit line to the material. If material is not included in the article's Creative Commons license and your intended use is not permitted by statutory regulation or exceeds the permitted use, you will need to obtain permission directly from the copyright holder. To view a copy of this license, visit http://creativecommons.org/ licenses/by/4.0/.

(c) The Author(s) 2021 